\title{
Neue Straflust der Strafjustiz - Realität oder Mythos?
}

Wolfgang Heinz

\section{Zunehmende Punitivität der deutschen Strafjustiz - Realität oder Mythos?}

Seit den 1980er Jahren hat in den USA ein „punitive turn“ der Kriminalpolitik stattgefunden. Die Gefangenenraten stiegen von 221 (1980) auf 758 (2008), haben sich also mehr als verdreifacht, ${ }^{1}$ die Strafzumessungsvorschriften für Rückfalltäter wurden drastisch verschärft, die Vollstreckung der Todesstrafe wurde in einigen Einzelstaaten wieder aufgenommen, Sexualstraftäter werden öffentlich bekannt gemacht und stigmatisiert. ${ }^{2}$ Auch in den meisten westeuropäischen Ländern sind die Gefangenenraten in den letzten 15 Jahren deutlich gestiegen; ${ }^{3}$ in Deutschland sind sie dagegen eher stabil geblieben. ${ }^{4}$ Dennoch wird auch hier von nicht wenigen Kriminologen eine „machtvolle Tendenz in Richtung auf Punitivität “" gesehen. Spätesten seit der zumindest verbalen Übernahme des englischen Tough on Crime-Ansatzes ${ }^{6}$ in Deutschland ${ }^{7}$ sowie der populistischen Parole „Wegschließen, und zwar für immer!“ "von Alt-Bundeskanzler Schröder ${ }^{8}$ wird befürchtet, es gebe keine „Kontinentalsperre“, die „den Strafvirus ab[halte], über Atlantik und Kanal zu uns herüber zu springen“. ${ }^{9}$ Die empirisch zu klärende Frage ist, ob die These einer zunehmenden Punitivität für Deutschland bestätigt werden kann.

\section{Indikatoren für zunehmende Punitivität und deren Messbarkeit}

Punitivität meint „mehr, härtere oder längere Strafen“. Aber „die“ Punitivität gibt es nicht. Attribute dieses Konstrukts können sich vielmehr beziehen auf

- die Forderung nach Strafverschärfungen im gesellschaftlichen Diskurs,

- die Erwartung härterer/längerer Strafen durch die Bürger,

- die Neukriminalisierung oder Verschärfung bestehender Strafvorschriften, insbesondere durch Erhöhung der Strafdrohungen,

- die Verhängung von mehr, härteren oder längeren Strafen durch die Gerichte sowie

- auf Verschärfungen bei der Strafvollstreckung.

Im folgenden Beitrag soll nur auf die Frage eingegangen werden, ob die These einer zunehmenden Punitivität der deutschen Strafjustiz empirisch bestätigt werden kann. In internationalen Vergleichen werden hierfür als Indikatoren zumeist nur Gefangenenraten verwendet oder (schon eher seltener) die Zugangsraten zum Strafvollzug, gemessen über die Zahl der Verurteilungen zu stationären Sanktionen. Damit wird freilich noch nicht einmal die Spitze des Eisbergs in den Blick genommen, denn in Deutschland machen stationäre Sanktionen - Jugendarrest, unbedingte Jugend- und Freiheitsstrafe sowie unbedingter Strafarrest - 8\% der Verurteilten und nur 3,4\% der (informell oder formell) Sanktionierten aus. Ein auch nur einigermaßen adäquates Bild der Sanktionierungspraxis als Grundlagen für deren Bewertung als mehr oder minder punitiv ist damit nicht zu gewinnen. Vielmehr muss die gesamte Sanktionierungspraxis in den Fokus genommen werden. Hierbei können die folgenden, aus der Punitivitätsthese ableitbaren Annahmen geprüft werden:

1. Es wird häufiger angeklagt und verurteilt, insbesondere

1.1 nimmt die Verurteiltenrate zu,

1.2 gehen die Raten der von der Staatsanwaltschaft gem. $\$ 170$ II StPO mangels hinreichenden Tatverdachts eingestellten Verfahren zugunsten von Einstellungen aus Opportunitätsgründen (=Diversion) oder von Anklagen sowie

1.3 die Einstellungen aus Opportunitätsgründen zugunsten von Anklagen zurück, ferner

1.4 nimmt innerhalb der Diversionsentscheidung der Anteil der unter Auflagen eingestellten Verfahren zu Lasten der folgenlosen Einstellungen zu.

2. Es wird häufiger Untersuchungshaft angeordnet.

3. Heranwachsende werden häufiger nach allgemeinem Strafrecht statt nach Jugendstrafrecht verurteilt.

4. Innerhalb der Verurteilungen

4.1 nimmt die Eingriffsintensität der ambulanten Sanktionen zu,

4.2 werden vermehrt stationäre statt ambulante Sanktionen verhängt,

4.3 werden Freiheitsstrafen seltener zur Bewährung ausgesetzt,

4.4 werden häufiger Freiheitsstrafen von längerer Dauer verhängt und

4.5 vermehrt freiheitsentziehende Maßregeln der Besserung und Sicherung angeordnet.

5. Die Strafvollstreckung wird „härter“, die Verlegungen in den offenen Vollzug gehen zurück, Strafrestaussetzungen werden seltener gewährt.

Aus Raumgründen kann im Folgenden nicht nach der Sanktionierungspraxis im Jugendstrafrecht einerseits, im allgemeinen Strafrecht andererseits differenziert werden; soweit ausgeprägt unterschiedliche Entwicklungen bestehen, werden diese textlich erwähnt. ${ }^{10}$

Die einzigen verfügbaren Datenquellen, um die genannten Annahmen prüfen zu können, bilden die Daten der Strafrechtspflegestatistiken. Diese sind freilich hinsichtlich einiger der Indikatoren zu wenig differenziert, lückenhaft und weisen immanente Verzerrungen auf. Insbesondere hinsichtlich der informellen Sanktionen, also den Opportunitätsentscheidungen, wird weder das Delikt noch werden Art und Schwere der angeordneten Auflage nachgewiesen. Weder bei den formellen, also den durch Urteil verhängten, noch den informellen Sanktionen ist eine etwaige Veränderung der Schwere der Tat, vom Straftatbestand bei den formell Sanktionierten abgesehen, messbar. Die Eingriffsintensität der formellen Sanktionen ist, wenn überhaupt, nur in Kategorien erfasst; deshalb ist z.B. eine durchschnittliche Strafdauer nicht ermittelbar 
und damit auch nicht deren Veränderung. Verurteilungen wegen mehrerer Straftaten sind nicht erkennbar, es wird immer nur das abstrakt schwerste Delikt nachgewiesen.

Unabhängig von diesem Problem unvollständiger und nicht hinreichend differenzierter Daten besteht das Problem, dass Effekte justizieller Selektion, hier: die Verschiebung der Grenze zwischen informeller (Diversion) und formeller Sanktionierung, nur begrenzt kontrollierbar sind. Dieses Messproblem wird regelmäßig nicht hinreichend erkannt, geschweige denn berücksichtigt. Angenommen, die Staatsanwaltschaften würden alle Ermittlungsverfahren einstellen, die mit großer Wahrscheinlichkeit nicht zu einer stationären Sanktion führen. Der Anteil der stationären Sanktionen an allen durch Urteil verhängten Sanktionen würde dementsprechend dramatisch steigen. Würde im Zeitverlauf, wie zumeist, nur die Veränderung der Anteile stationärer Sanktionen an den Verurteilten berücksichtigt, dann müsste eine entsprechend dramatische Zunahme von Punitivität abgeleitet werden. Diese Folgerung wäre indes falsch, denn der Anstieg der Rate stationärer Sanktionen wäre ja nur Folge einer Ausfilterung leichter und mittelschwerer Kriminalität, beruhte also auf einer Verschiebung der Grenze zwischen Diversion und Verurteilung. Die Grundgesamtheit, auf die Bezug genommen werden muss, sind nicht die Verurteilten, sondern die (informell und formell) Sanktionierten. Dieses Gedankenexperiment ist überspitzt, gleichwohl ist es nicht realitätsfremd. Denn zwischen 1981 und 2008 ist die Diversionsrate von 36\% auf 58\% gestiegen (vgl. Schaubild 1). Hierdurch dürften zunehmend leichte und mittelschwere Formen der Kriminalität aus dem Bereich der formellen Sanktionen ausgefiltert worden sein. Genau weiß man dies freilich nicht, weil die Daten der StA-Statistik zu den Einstellungen keine Straftatbestände ausweisen. Aus empirischen Untersuchungen ist aber bekannt, dass Diversion sich inzwischen auf Fallgruppen erstreckt, die noch vor einigen Jahren zur Verurteilung geführt hätten, weshalb diese Annahme sehr viel empirische Plausibilität für sich hat. ${ }^{11}$

Dieses Messproblem besteht sowohl bei zeitlichen Längsschnittvergleichen als auch bei Querschnittsvergleichen. Gleiche Höhe oder Stabilität der Diversionsraten werden nämlich regelmäßig nicht bestehen. Dass und wie sehr sich Unterschiede in der Höhe informeller Sanktionen den Vergleich verzerren können, zeigen aktuelle Daten. Bei Berechnung der Rate stationärer Sanktionen (unbedingte Jugend- oder Freiheitsstrafe), bezogen auf Verurteilte, nimmt Baden-Württemberg 2008 auf einer Rangskala von wenig bis stark punitiv Platz 6 ein, bezogen dagegen auf Sanktionierte nur noch Platz 13. Daraus folgt, dass ohne Berücksichtigung informeller Sanktionen die Raten formell Verurteilter weder im nationalen Querschnitt noch im Längsschnitt zutreffend eingeordnet und bewertet werden können. Dasselbe gilt für den internationalen Vergleich. Umgehen lässt sich dieses Messproblem nur dadurch, dass die Analyse auf Schwerkriminalität beschränkt wird, bei der Diversion keine Rolle spielt; dies geht freilich zulasten der Verallgemeinerungsfähigkeit der Befunde.

Wegen der leichteren Verfügbarkeit werden international vor allem die Gefangenenraten, also die Zahl der Gefangenen pro 100.000 der (strafmündigen) Wohnbevölkerung, als Punitivitätsindikator herangezogen. Unter allen in Betracht kommenden Indikatoren sind aber Gefangenenraten hierfür am wenigsten geeignet, abgesehen davon, dass es sich um die Spitze der Spitze des Eisberges handelt. Denn Gefangenenraten sind, im Vergleich zu den Daten über Verurteilte, mit zusätzlichen Problemen behaftet: ${ }^{12}$

- Es handelt sich (jedenfalls in Deutschland) um Stichtagsdaten. Stichtagsdaten sind aber eine Funktion der Zahl der Zugänge und der Verweildauer in den Anstalten. Gefangenenraten zeigen also eine Ausweitung der Zahl der Verurteilungen (mehr Strafen) oder eine Verlängerung der durchschnittlichen Haftdauer (sei es durch die Verhängung von Strafen mit längerer Dauer, sei es durch Einschränkungen der vorzeitigen Entlassung) oder zeigen eine Kombination von beiden an. Ein „Mehr“ an Zugängen aufgrund von unbedingt verhängten Strafen ist aber nicht gleichbedeutend mit zunehmender Punitivität. Denn bei steigendem Input (als Folge gestiegener „Kriminalität“) kommt es auch bei unveränderter Strafzumessungspraxis zu einem „Mehr“ an Zugängen in den Strafvollzug.

- Ein Teil der Gefangenen ist nicht primär zu einer Freiheitsstrafe verurteilt, sondern verbüßt eine Freiheitsstrafe als sog. „Ersatzfreiheitsstrafe“ wegen einer nicht beitreibbaren Geldstrafe oder wegen Widerrufs einer zur Bewährung ausgesetzten Freiheitsstrafe. Beide Fallgruppen sind - sofern sie nicht auf einer Änderung der Vollstreckungspraxis beruhen - kein Indiz für zunehmende Punitivität.

- Hinzu kommt schließlich (in Deutschland) noch ein Schätzfehler durch unvollständige Bevölkerungszahlen. ${ }^{13}$ Dieser Fehler dürfte in den letzten Jahren aufgrund von Migrationsbewegungen deutlich größer geworden sein mit der Folge einer zunehmenden Überschätzung der Gefangenenraten.

Die Zunahme polizeilich registrierter Kriminalität ist kein Indikator für justizielle Punitivität. Umfang, Struktur und Entwicklung der polizeilich registrierten Kriminalität werden fast völlig vom Anzeigeerstatter bestimmt. Deshalb handelt es sich hier allenfalls (bei unveränderter „Kriminalitätswirklichkeit“) um zunehmende gesellschaftliche Punitivität. Ebenso wenig wird die polizeiliche Aufklärungstätigkeit und die hierbei erfolgende Schwerpunktsetzung als Indikator justizieller Punitivität gewertet werden können. Deren Verarbeitung durch strafrechtliche Sozialkontrolle ist dann, aber auch nur dann justiziell zunehmende Punitivität, wenn die Reaktionsmaßstäbe verschärft werden.

\section{Zunehmende Punitivität der Strafjustiz - die Befunde}

\subsection{Zunehmende Punitivität durch ein „Mehr“ an Sanktionierung?}

Zumindest zwei Ausprägungen dieses Indikators können unterschieden werden:

- Anteil der (informell oder formell) Sanktionierten an den polizeilich ermittelten Tatverdächtigen.

- Anteil der mangels hinreichenden Tatverdachts von der Staatsanwaltschaft (StA) eingestellten Ermittlungsverfahren an allen anklagefähigen oder gem. $\$ 170$ II StPO eingestellten Ermittlungsverfahren (= mehr staatsanwaltliche Intervention).

Das deutsche kriminalstatistische System erlaubt wegen unterschiedlicher Erfassungsgrundsätze und -zeiträume, Erhebungseinheiten und Zählweisen keinen direkten Vergleich zwischen den Aggregatdaten der Tatverdächtigen und der Sanktionierten. Eingeschränkt möglich ist lediglich die Gegenüberstellung von Tatverdächtigen und Verurteilten. Diese geben keinen Hinweis auf zunehmende Punitivität, denn die Relationen Tatverdächtige : Verurteilte sind tendenziell rückläufig. ${ }^{14}$ 
Entgegen der Punitivitätsthese sind aber auch die Anteile von $\$ 170$ II StPO im Wesentlichen konstant geblieben. Die Ausweitung der Opportunitätseinstellungen ging nicht zu Lasten der Einstellungen mangels hinreichenden Tatverdachts, sondern - aus Sicht der Punitivitätsthese erwartungswidrig - zu Lasten von Anklagen bzw. Strafbefehlsanträgen. ${ }^{15}$

\subsection{Zunehmende Punitivität durch ein „Mehr“ an intervenierender Diversion und/oder ein „Mehr“ an formeller} Sanktionierung?

Auf der Ebene der staatsanwaltlichen Entscheidungen wäre bei zunehmender Punitivität zu erwarten, dass

- innerhalb der Opportunitätseinstellungen der Anteil der unter Auflagen eingestellten Verfahren zu Lasten der folgenlosen Einstellungen zunimmt,

- die Einstellungen aus Opportunitätsgründen zugunsten von Anklagen zurückgehen.

Schaubild 1 zeigt, dass beide Annahmen empirisch nicht bestätigt werden können. Die Anteile der Einstellungen unter Auflagen sind zurückgegangen, und zwar sowohl bezogen auf Sanktionierte insgesamt als auch an den Opportunitätseinstellungen selbst. Die Opportunitätseinstellungen sind ebenfalls nicht zugunsten von formeller Sanktionierung zurückgegangen, sie sind vielmehr deutlich gestiegen, seit einigen Jahren stagnieren sie auf hohem Niveau.

Mangels Daten in den Strafrechtspflegestatistiken kann allerdings nicht geprüft werden, ob innerhalb der Formen intervenierender Diversion die Intensität der Auflagen zugenommen hat.

Schaubild 1: Informell und formell Sanktionierte. Anteile. Früheres Bundesgebiet mit Westberlin, seit 1995 mit Gesamtberlin (FG), seit 2007 FG und Deutschland

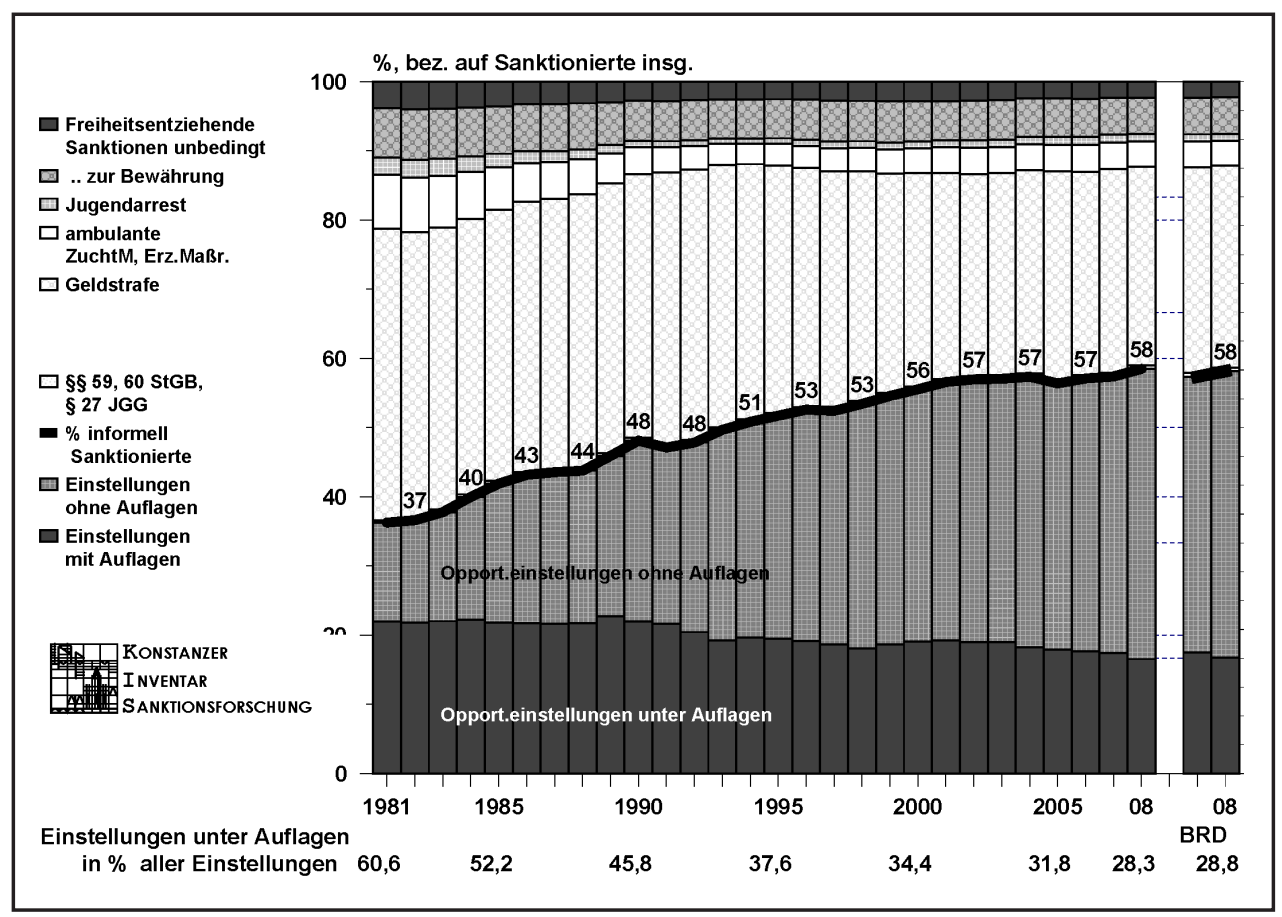

Informell Sanktionierte (Opportunitätseinstellungen): §§ 153, 153a, 153b StPO, §§ 45, 47 JGG; §§ 31a, 37 BtMG.

Opportunitätseinstellungen unter Auflagen: § 153a StPO, §§ 45 III, 47 JGG; § 37 BtMG.

Datenquellen: Staatsanwaltschaftsstatistik, Strafverfolgungsstatistik

\subsection{Zunehmende Punitivität durch häufigere Anordnung von Untersuchungshaft?}

Bei zunehmender Punitivität wäre zu erwarten, dass häufiger Untersuchungshaft (U-Haft) angeordnet wird. ${ }^{16}$ Um den verzerrenden Einfluss von Diversion zu vermeiden, werden im Folgenden nur diejenigen Delikte berücksichtigt, die zum einen (mutmaßlich) nicht von Diversionsentscheidungen betroffen sind, auf die zum anderen ein großer Teil der U-Haftanordnungen entfällt. Hier zeigt sich, dass

- in den letzten 35 Jahren weder bei vorsätzlichen Tötungs- noch bei Raubdelikten, weder bei gefährlicher Körperverletzung noch bei Einbruchsdiebstahl der Anteil der Verurteilten mit vorangegangener Untersuchungshaft kontinuierlich und nachhaltig angestiegen ist (vgl. Schaubild 2).

- Bei Einbruchdiebstahl gab es seit Ende der 1980er Jahre einen deutlichen Anstieg. Deutlich abgeschwächt ist dies auch bei den anderen Straftaten festzustellen. Seit 2004 gehen die Raten indes wieder zurück und sind seitdem auf einem Tiefstand.

- 2008 sind die U-Haftraten bei allen hier geprüften Deliktsgruppen wieder gestiegen, und zwar sowohl in den alten wie in den neuen Ländern; es bleibt abzuwarten, ob dies eine gegenläufige Tendenz einleitet. 
Schaubild 2: Wegen schwerer Delikte Verurteilte mit vorangegangener Untersuchungshaft. Anteile bezogen auf die jeweilige Gesamtzahl der Verurteilten. Früheres Bundesgebiet mit Westberlin, seit 1995 mit Gesamtberlin (FG), seit 2007 Deutschland

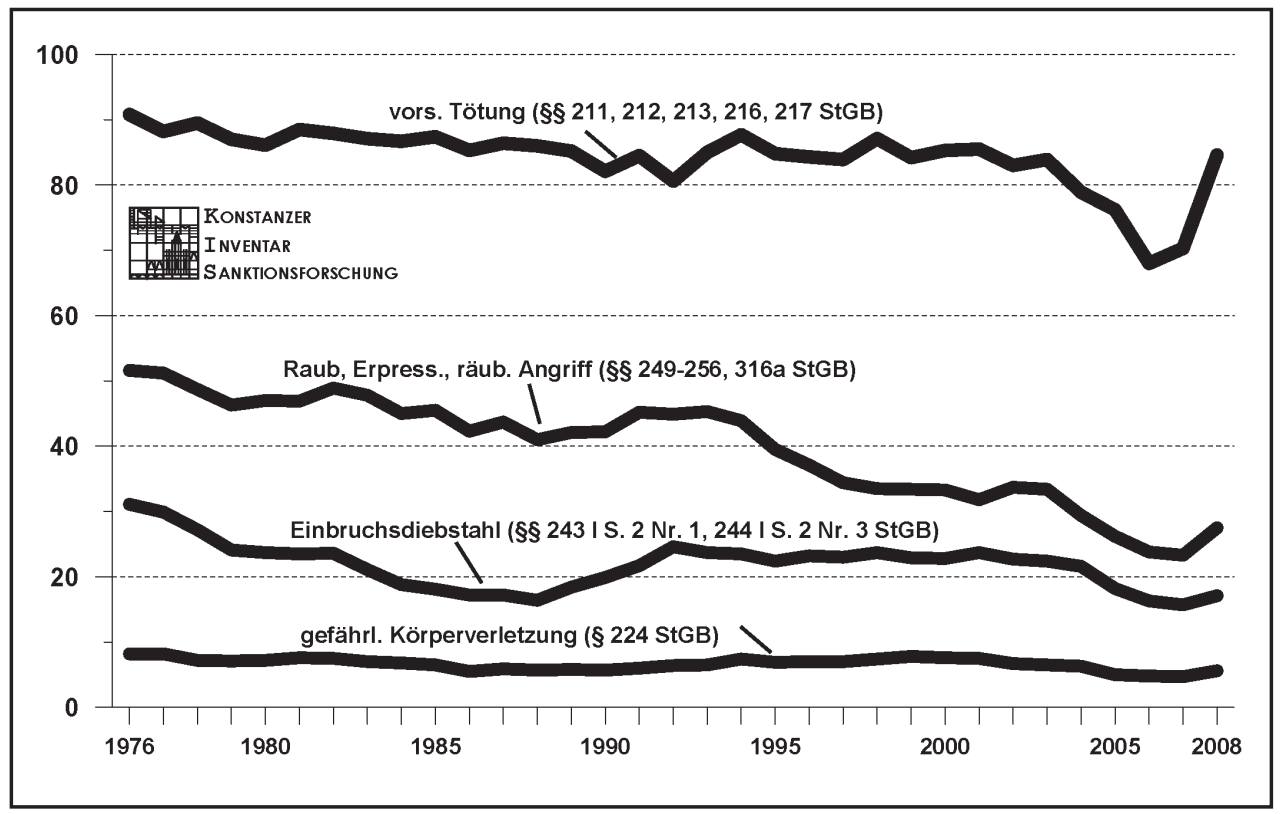

Datenquellen: Strafverfolgungsstatistik

\subsection{Zunehmende Punitivität durch häufigere Einbeziehung von Heranwachsenden in das allgemeine Strafrecht?}

Seit vielen Jahren wird von Teilen der Politik die Forderung erhoben, auf Heranwachsende in der Regel wieder das allgemeine Strafrecht anzuwenden. Die derzeitige Regelanwendung des Jugendstrafrechts lasse, so wird argumentiert, bei schweren Formen der Kriminalität keine schuldangemessene Ahndung zu. Bei Einbeziehung in das allgemeine Strafrecht sei die Strafobergrenze höher (15 Jahre Freiheitsstrafe statt 10 Jahre Jugendstrafe), es könne eine lebenslange Freiheitsstrafe verhängt und eher eine Sicherungsverwahrung angeordnet werden. Bei zunehmender Punitivität der Jugendgerichte wäre deshalb zu erwarten, dass wieder vermehrt allgemeines Strafrecht angewendet wird.

Entgegen der Punitivitätsthese ist insgesamt keine Trendwende festzustellen. Die Einbeziehensrate in das allgemeine Strafrecht war zwar in der ersten Hälfte der 1990er Jahre leicht rückläufig, was vor allem auf einer Sonderentwicklung bei nichtdeutschen Heranwachsenden beruhte, insgesamt aber blieb das hohe Niveau erhalten. ${ }^{17} 2008$ war in den alten Ländern sogar die höchste, jemals gemessene Einbeziehensrate erreicht.

\subsection{Zunehmende Punitivität durch ein „Mehr“ an härteren oder längeren formellen Sanktionen?}

Auf der Ebene der gerichtlichen Entscheidungen wäre zu erwarten, dass

- vermehrt verurteilt statt aus Subsidiaritätsgründen eingestellt wird,

- die Eingriffsintensität der ambulanten Sanktionen zunimmt,

- häufiger stationäre statt ambulante Sanktionen verhängt werden,

- verhängte Jugend- oder Freiheitsstrafen seltener zur Bewährung ausgesetzt werden,

- vermehrt stationäre Sanktionen von längerer Dauer verhängt werden.

Schaubild 1 hat bereits gezeigt, dass die Annahme der Punitivitätsthese nicht bestätigt werden kann, es werde vermehrt verurteilt statt aus Subsidiaritätsgründen eingestellt. Die Rate der formell Sanktionierten ist von $64 \%$ auf $42 \%$ zurückgegangen. In den letzten 10 Jahren blieb sie auf diesem hohen Niveau unverändert.

Die weiteren Thesen sind anhand der Daten der Strafrechtspflegestatistiken nur begrenzt prüfbar. Dies beruht auf der Zunahme der Diversionsentscheidungen, durch die mutmaßlich vermehrt leichte Formen der Kriminalität ausgefiltert werden mit der Konsequenz, dass unter den Verurteilten der Anteil schwerer Formen der Kriminalität zugenommen haben dürfte, die eher mit stationären Sanktionen geahndet werden.

Am Beispiel der stationären Sanktionen soll dieses Messproblem und seine Folgen verdeutlicht werden (vgl. Schaubild 3). Die Raten stationärer Sanktionen ${ }^{18}$ - bezogen auf Verurteilte - sind langfristig rückläufig. Der aus dem Schaubild ersichtliche Rückgang stationärer Sanktionen 1969 ist Folge der damaligen Sanktionenrechtsreform, durch die im allgemeinen Strafrecht die kurze Freiheitsstrafe unter 6 Monaten zurückgedrängt und der Anwendungsbereich der Strafaussetzung zur Bewährung von 9 auf 24 Monate erweitert wurde. Seit 1993 nehmen die stationären Sanktionen indes wieder zu, was als zunehmende Punitivität gedeutet werden könnte. Zwischen 1993 und 2008 stieg freilich auch die Diversionsrate von 49,7\% auf 58,2\%. Die Auswirkungen zunehmender Diversion können indirekt kontrolliert werden, indem die stationären Sanktionen auf Sanktionierte bezogen wird. Diese Rate stieg - im Unterschied zu der auf Verurteilte bezogenen Rate - seit 1993 kaum an (1993: 3,4\%, 2003: 3,8\%; 2008: 3,4\%), so dass sich von daher kein Hinweis auf zunehmende Punitivität ableiten lässt. 
Schaubild 3: Nach allgemeinem und nach Jugendstrafrecht zu stationären Sanktionen (unbedingte Freiheits-/Jugendstrafe, unbed. Strafarrest, Jugendarrest) Verurteilte (Anteile bezogen auf Verurteilte bzw. Sanktionierte) und Diversionsrate. Früheres Bundesgebiet mit Westberlin, seit 1995 mit Gesamtberlin, seit 2007 Deutschland

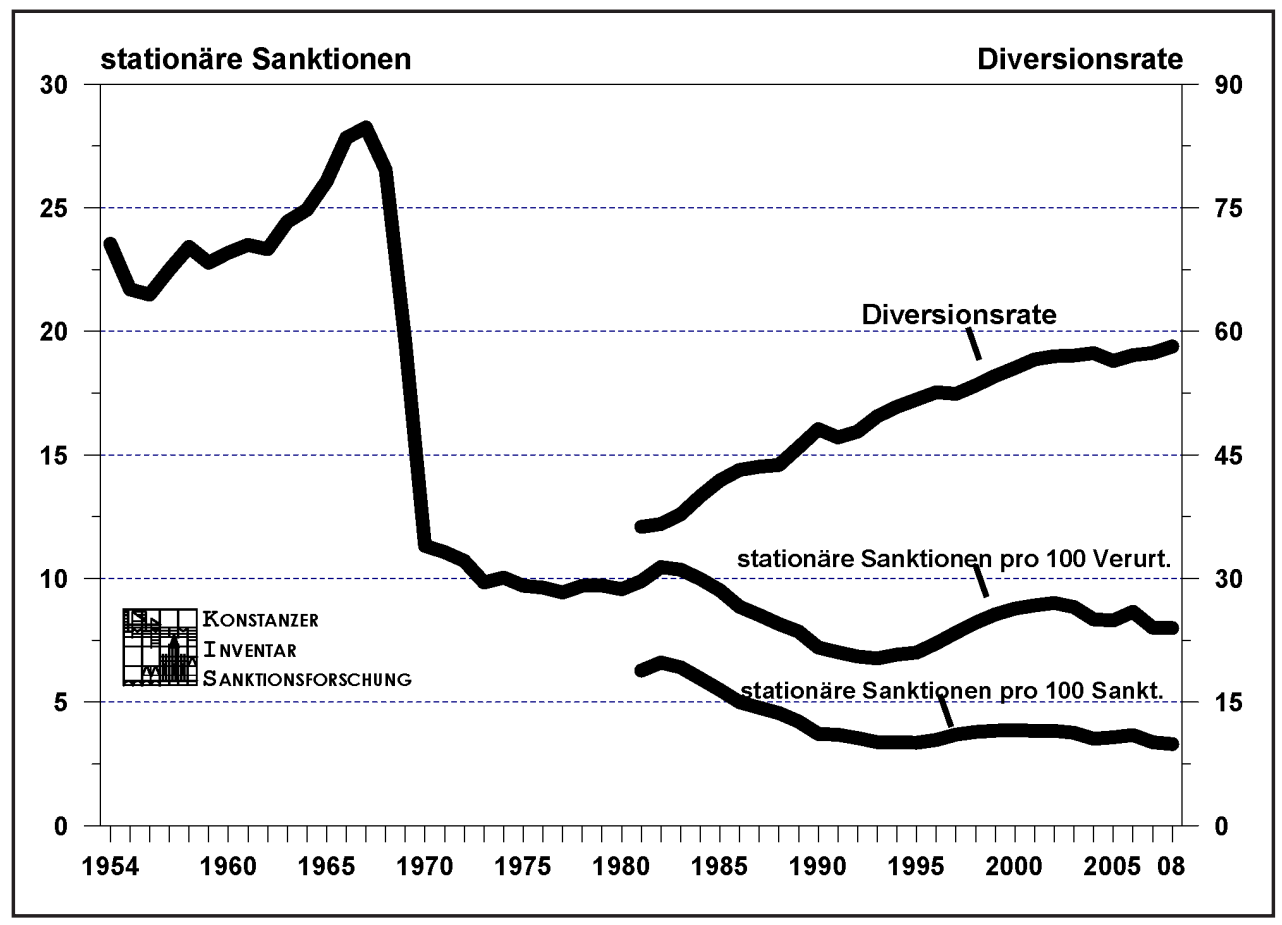

Datenquellen: Staatsanwaltschaftsstatistik, Strafverfolgungsstatistik.

Hinsichtlich der Eingriffsintensität ambulanter Sanktionen enthält die StVerfStat lediglich Angaben zur Geldstrafe; Daten zur Höhe der gem. \15 JGG erteilten Auflagen oder zu den im Zusammenhang mit einer Strafaussetzung zur Bewährung erteilten Weisungen oder Auflagen werden nicht erhoben.

Bezogen auf nach allgemeinem Strafrecht Verurteilte ist zwar der Anteil der zu Geldstrafe Verurteilten im Wesentlichen unverändert geblieben, die Zahl der verhängten Tagessätze ${ }^{19}$ stieg dagegen deutlich an. 1981 wurden 25,9\% der Verurteilten zu 31 oder mehr Tagessätzen verurteilt, 2008 waren es dagegen 43,4\%. Wird freilich berücksichtigt, dass durch vermehrte Diversion mutmaßlich immer mehr Bagatellfälle nicht mehr zur Verurteilung gelangen, dann relativiert sich dieses Bild. Denn bezogen auf Sanktionierte ergibt sich nur ein geringer Zuwachs von $17 \%$ auf $19,6 \%$. Hinzu kommt, dass nur die Veränderung von Kategorien gemessen werden kann, nicht aber die eigentlich relevante durchschnittliche Höhe der Zahl der Tagessätze.

Entsprechend der Punitivitätsthese wäre ferner zu erwarten, dass verhängte Freiheits- oder Jugendstrafen seltener zur Bewährung ausgesetzt werden. Die Raten der zur Bewährung ausgesetzten Strafen sind jedoch - entgegen der Punitivitätsthese - nicht zurückgegangen (vgl. Schaubild 4).

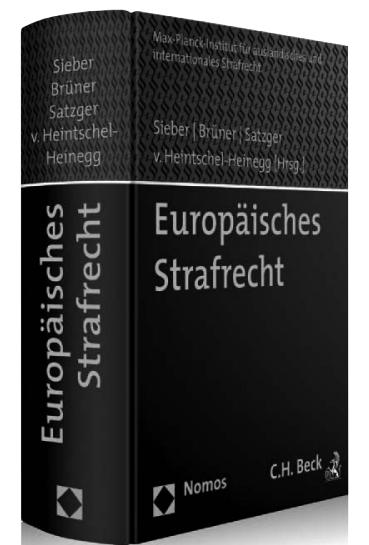

\section{Europäisches Strafrecht}

Herausgegeben vom Max-Planck-Institut für ausländisches und internationales Strafrecht, durch Prof. Dr. Dr. h.c. Ulrich Sieber, in Zusammenarbeit mit Gen.-Dir. Franz-Hermann Brüner †, Prof. Dr. Helmut Satzger und Prof. Dr. Bernd von Heintschel-Heinegg, VRiBayOLG

2011, ca. 800 S., geb., 148,-€, ISBN 978-3-8329-5603-5

Erscheint ca. März 2011

Mit dem Handbuch zum Europäischen Strafrecht ist unter Federführung des Max-Planck-Instituts für ausländisches und internationales Strafecht und in Herausgeberschaft führender Strafrechtswissenschaftler ein fundiertes wie souverän geschriebenes Werk entstanden, das übersichtlich wie verständlich keine Fragen offen lässt. 
Schaubild 4: Aussetzungsraten. Zur Bewährung ausgesetzte Freiheits- oder Jugendstrafen bezogen auf aussetzungsfähige Strafen der jeweiligen Kategorie (Aussetzungsraten). Früheres Bundesgebiet mit Westberlin, seit 1995 mit Gesamtberlin (FG), seit 2007 Deutschland

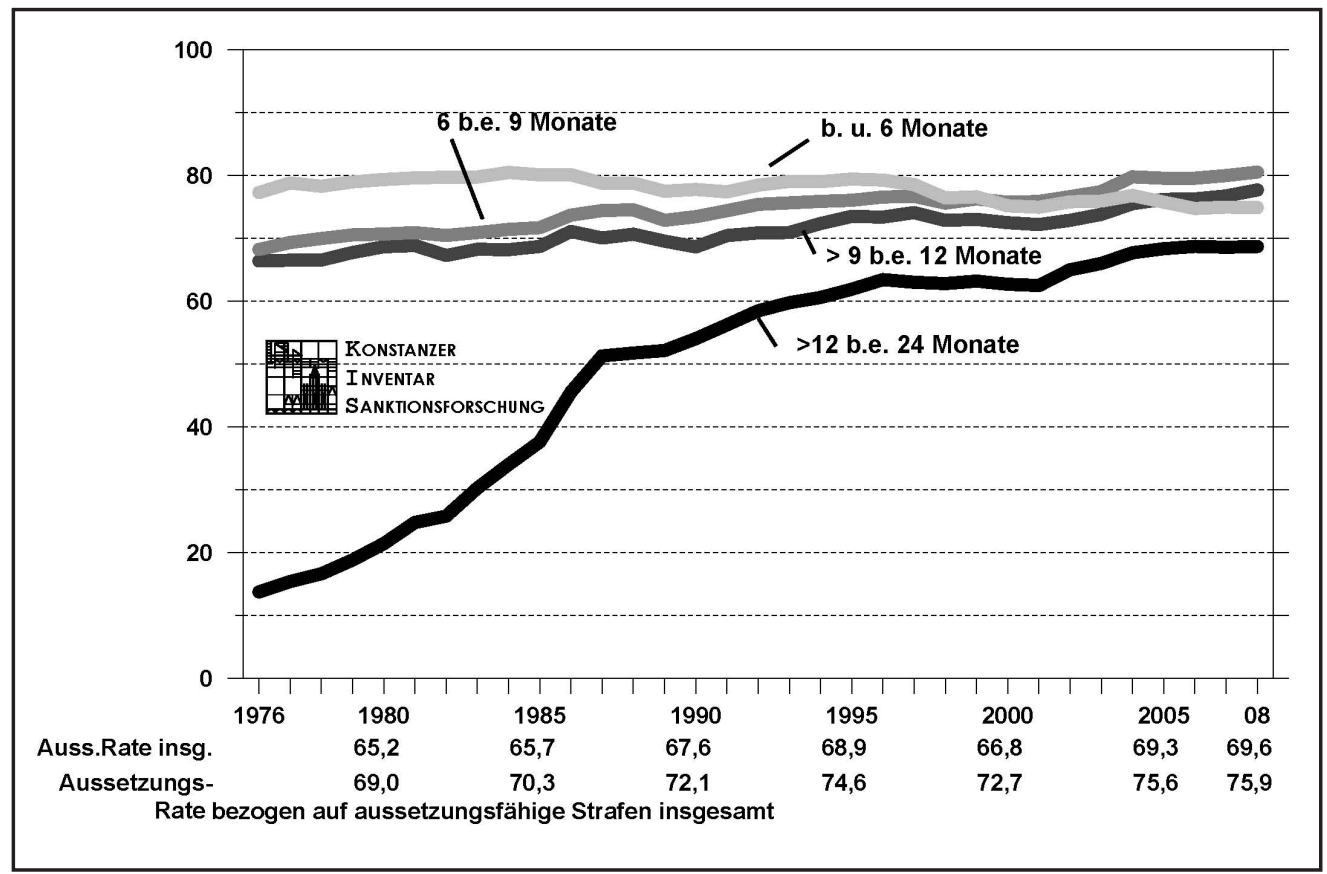

Datenquellen: Strafverfolgungsstatistik.

Dass insgesamt relativ mehr Freiheits- und Jugendstrafen sowie Jugendarreste verhängt werden, ist eine weitere, aus der Punitivitätsthese ableitbare Annahme. Schaubild 5 zeigt, dass es in den letzten knapp 40 Jahren nach der Strafrechtsreform von 1969 Wellenbewegungen gegeben hat. Ein erste Anstiegswelle war zwischen 1980 und 1984 zu beobachten, eine zweite zwischen 1992 und 2002 . Seitdem sind die Raten der insgesamt verhängten Freiheits- und Jugendstrafen wieder zurückgegangen; sie liegen derzeit auf dem Niveau von 1984. Weitgehend aufgefangen wurde dieser Anstieg durch zunehmend erfolgte Strafaussetzung zur Bewährung. Die unbedingt verhängten Freiheits- und Jugendstrafen stiegen nur geringfügig. 2008 sind sie unter dem Niveau der 1970er und fast der gesamten 1980er Jahre. Werden die Raten auf die Sanktionierten bezogen, dann wären sie derzeit sogar auf dem niedrigsten, jemals gemessenen Stand.

Schaubild 5: Zu Freiheits- oder Jugendstrafe sowie zu Jugendarrest Verurteilte. Anteile bezogen auf Verurteilte. Früheres Bundesgebiet mit Westberlin, seit 1995 mit Gesamtberlin (FG), seit 2007 Deutschland

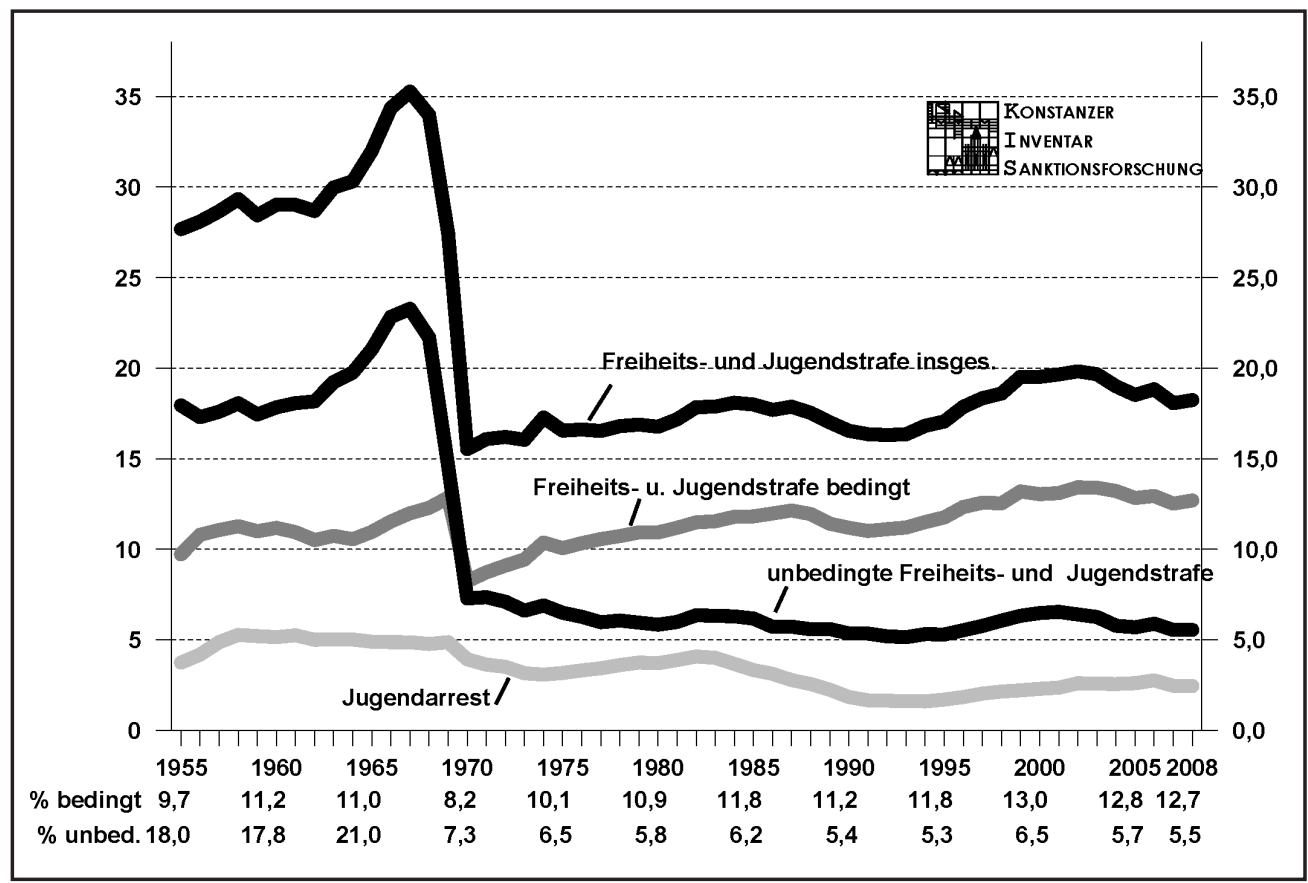

Datenquellen: Strafverfolgungsstatistik.

Schließlich wäre zu erwarten, dass vermehrt stationäre Sanktionen von längerer Dauer verhängt werden. Innerhalb der stationären Sanktionen kam es in der Tat zu Verschiebungen in Richtung auf Freiheitsstrafen von längerer Dauer (vgl. Schaubild 6). Dies beruht vor allem auf 
nach allgemeinem Strafrecht verhängten unbedingten Freiheitsstrafen zwischen 2 und 5 Jahren. Im Jugendstrafrecht ist der Anteil unbedingt verhängter Jugendstrafen sowohl im Bereich der Jugendstrafen zwischen 1 und 2 Jahren als auch zwischen 2 und 3 Jahren gestiegen, der Anteil der längerfristigen Jugendstrafen blieb dagegen unverändert.

Schaubild 6: Unbedingt verhängte Freiheits- oder Jugendstrafen nach der Dauer. Anteile bezogen auf die Verurteilte. Früheres Bundesgebiet mit Westberlin, seit 1995 mit Gesamtberlin, seit 2007 Deutschland

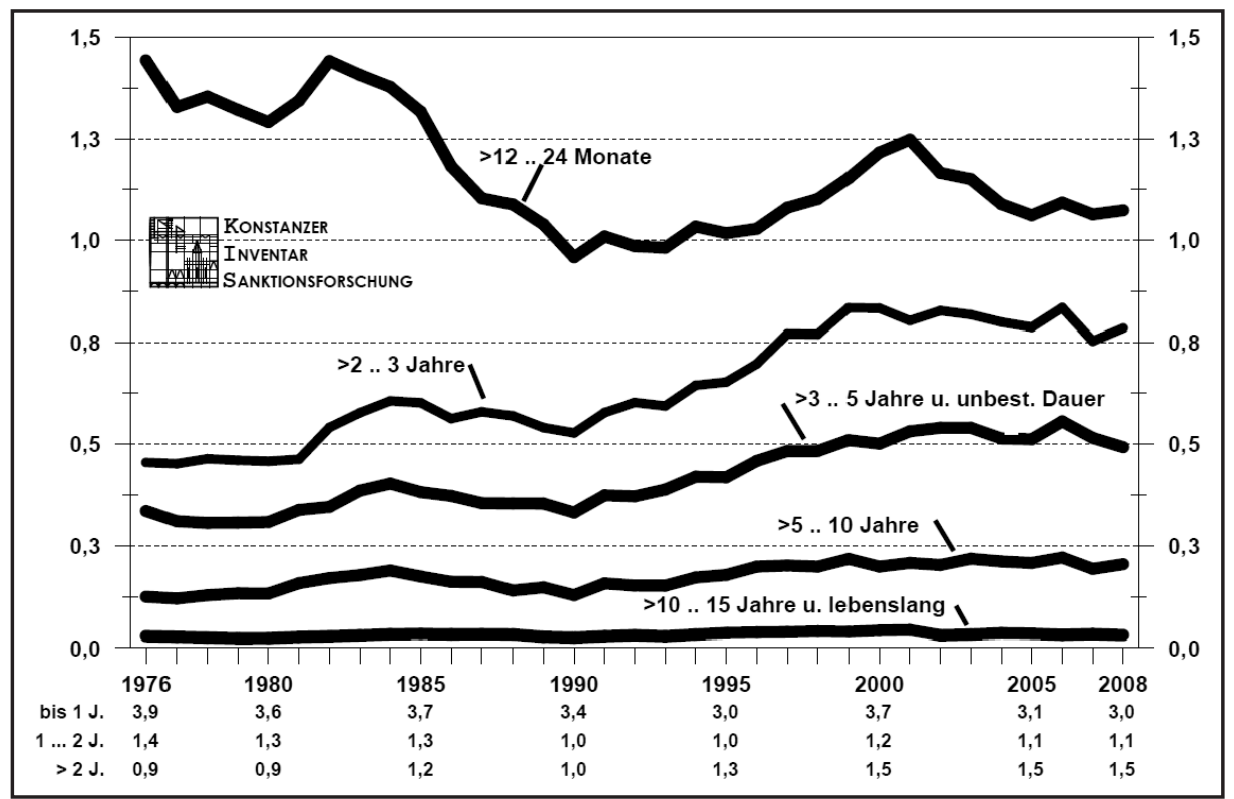

Datenquellen: Strafverfolgungsstatistik.

Wird freilich der verzerrende Einfluss zunehmender Diversionsentscheidungen berücksichtigt, indem die Anteile nicht auf Verurteilten, sondern auf die Sanktionierten bezogen werden, dann ist zwischen 1990 und 2008 lediglich bei den unbedingt verhängten Freiheits- oder Jugendstrafen von mehr als zwei Jahren eine Zunahme um 0,11 Prozentpunkte festzustellen (vgl. Schaubild 7).

Hinweise auf zunehmende Punitivität gibt es demnach bei einer Aggregatdatenanalyse der Strafrechtspflegestatistiken nicht hinsichtlich der Art der verhängten Sanktionen, sondern nur hinsichtlich einer wenig stark ausgeprägten Erhöhung des Strafenniveaus vor allem der stationären Sanktionen. Hier hängt das Ergebnis aber ersichtlich davon ab, wie der Einfluss zunehmender Diversion eingeordnet und bewertet wird. Eine eindeutige Aussage dürfte bei deliktspezifischer Betrachtung solcher Delikte zu erwarten sein, bei denen eine Verzerrung durch Diversion vernachlässigbar gering sein dürfte - vorsätzliche Tötungsdelikte, sexuelle Nötigung/Vergewaltigung, gefährliche Körperverletzung, Einbruchsdiebstahl und Raub.

Schaubild 7: Unbedingt verhängte Freiheits- oder Jugendstrafen nach der Dauer. Anteile bezogen auf Sanktionierte. Früheres Bundesgebiet mit Westberlin, seit 1995 mit Gesamtberlin, seit 2007 Deutschland

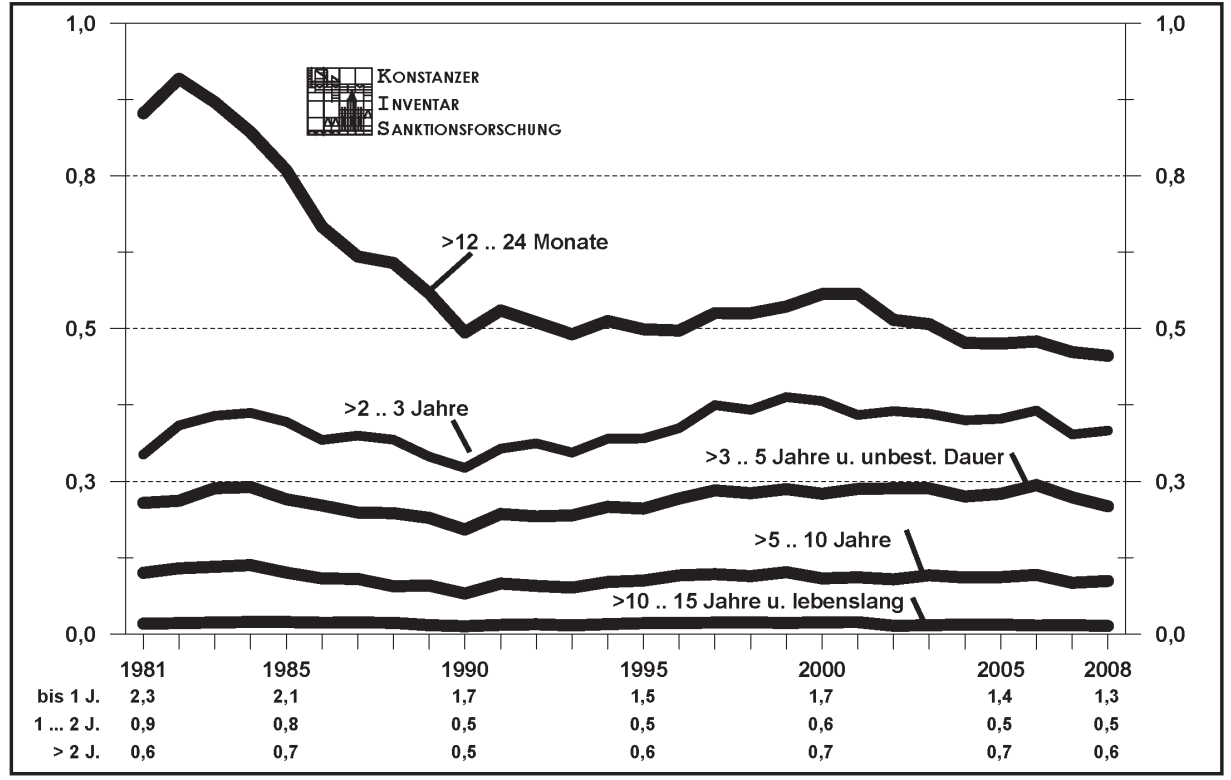

Datenquellen: Staatsanwaltschaftsstatistik, Strafverfolgungsstatistik 
Bei vorsätzlichen Tötungsdelikten (vgl. Schaubild 8) zeigt sich:

- Zu über 90\% werden unbedingte Freiheits- oder Jugendstrafen verhängt. Deren Anteil ist seit Anfang der 1990er Jahre gestiegen.

- Das Strafenniveau hat sich erhöht. Zugenommen hat vor allem der Anteil der lebenslangen Freiheitsstrafen sowie - in geringerem Maße - der Anteil der Freiheitsstrafen von mehr als 5 bis 10 Jahren. Diese Zunahme ging vor allem zu Lasten der Freiheitsstrafen unter 3 Jahren.

- Diese Verschiebung des Strafenniveaus ging einher mit einem Rückgang des Anteils der vermindert schuldfähigen Abgeurteilten, deren Strafe gem. S S 21, 49 StGB gemildert werden kann, und zwar bei Mord von 30\% auf $11 \%$, bei Totschlag von $41 \%$ auf $24 \% .{ }^{20}$ Auf der Grundlage der statistischen Daten lässt sich nicht entscheiden, ob sich die Beurteilung der Sachverständigen geändert hat oder aber die Sachverhalte.

Schaubild 8: Wegen vorsätzlicher Tötungsdelikte $(\mathbb{S} \mathbb{2} 211,212,213$ StGB) zu Freiheits- oder Jugendstrafe Verurteilte nach der Strafdauer sowie Strafaussetzung zur Bewährung. Anteile bezogen auf Verurteilte. Früheres Bundesgebiet mit Westberlin, seit 2005 mit Gesamtberlin, seit 2007 Deutschland

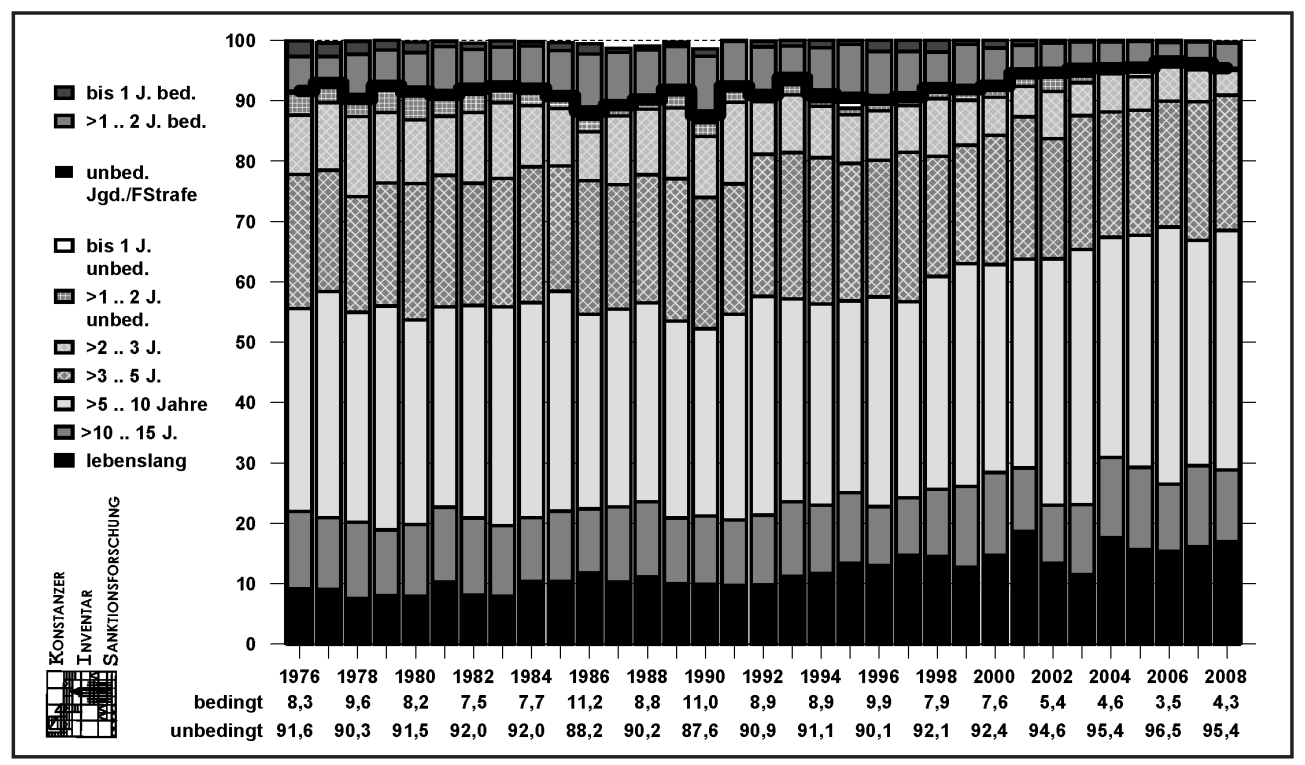

Datenquellen: Strafverfolgungsstatistik.

Die 1998 erfolgte straftatbestandliche Ausweitung bei $\$ 177$ StGB beeinträchtigt zwar den Vergleich; die Daten zeigen aber (vgl. Schaubild 9) weder eine Zunahme der unbedingten Freiheits- und Jugendstrafen noch eine durchgängige Erhöhung des Strafenniveaus. ${ }^{21}$ Das Strafenniveau hat sich bis 1998 erhöht, seitdem bleibt der Anteil der Strafen von mehr als 3 Jahren unverändert, unbedingte Strafen bis 3 Jahre werden relativ seltener ausgesprochen. Innerhalb der bedingten Freiheits- und Jugendstrafen kam es zu einem Austausch zwischen Strafen bis 1 Jahr und den Strafen zwischen 1 Jahr und 2 Jahren.

Schaubild 9: Wegen sexueller Nötigung / Vergewaltigung (bis $1997 \rrbracket 177$ Abs. 1 StGB, seit 1999 mit $\ 178$ StGB) zu Jugendarrest oder Jugendstrafe (insgesamt) Verurteilte nach der Strafdauer. Anteile bezogen auf Verurteilte. Früheres Bundesgebiet mit Westberlin, seit 2005 mit Gesamtberlin, seit 2007 Deutschland

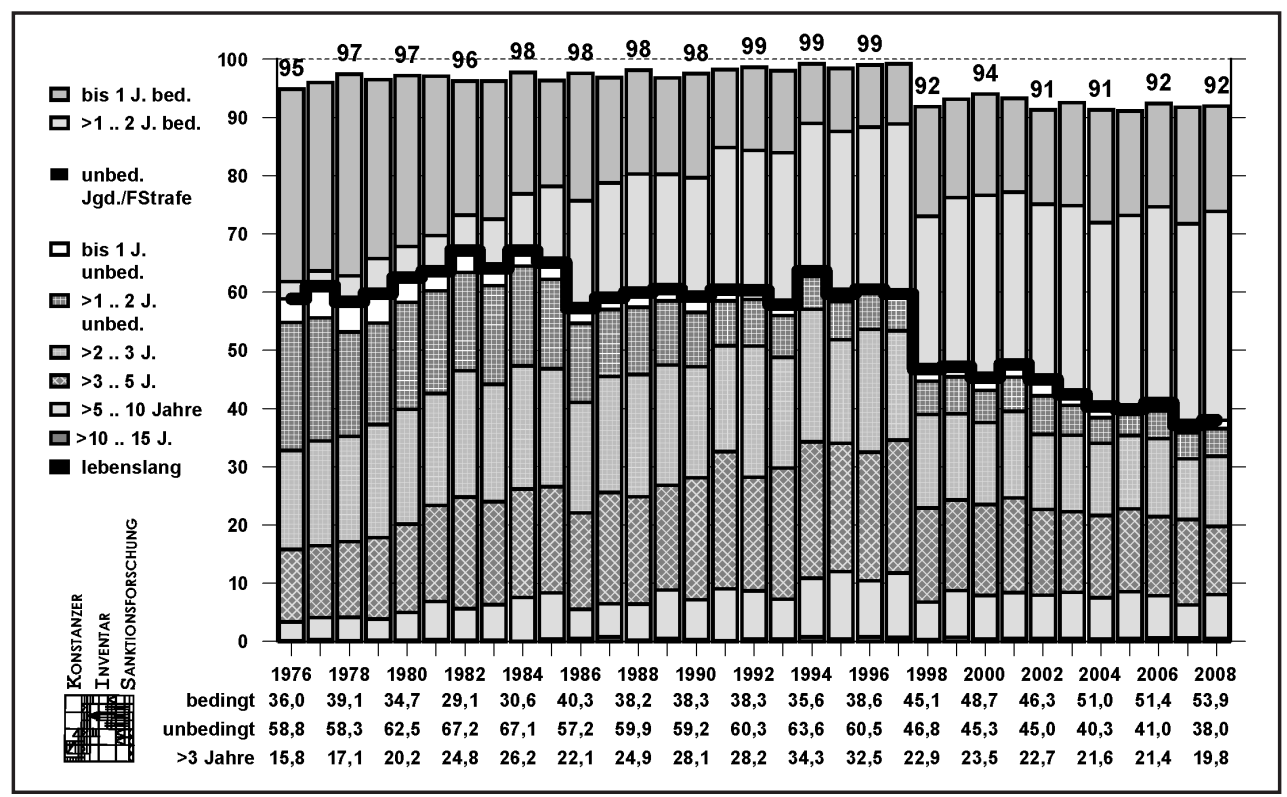

Datenquellen: Strafverfolgungsstatistik. 
Im Unterschied zu den anderen, in die Betrachtung einbezogenen Delikten zeigt sich bei gefährlicher Körperverletzung ( $\$ 224$ StGB) eine deutlich unterschiedliche Entwicklung der Sanktionierungspraxis im allgemeinen Strafrecht einerseits, im Jugendstrafrecht andererseits. Im allgemeinen Strafrecht (vgl. Schaubild 10)

- hat sich der Anteil der insgesamt verhängten Freiheitsstrafen mehr als verdoppelt, wobei diese Zunahme fast ausschließlich im Bereich der ausgesetzten Freiheitsstrafen erfolgte und hier vor allem bei Freiheitsstrafen bis 1 Jahr,

- sind sowohl der Anteil der unbedingt verhängten Freiheitsstrafen als auch das Strafenniveau innerhalb der unbedingten Freiheitsstrafen nach einer zunächst leicht rückläufigen Entwicklung seit Mitte der 1990er Jahre wieder bis 2001 gestiegen.

Schaubild 10: Wegen gefährlicher Körperverletzung $(\$ 224$ StGB) zu Freiheitsstrafe Verurteilte nach der Strafdauer sowie Strafaussetzung zur Bewährung. Anteile bezogen auf Verurteilte. Früheres Bundesgebiet mit Westberlin, seit 2005 mit Gesamtberlin, seit 2007 Deutschland

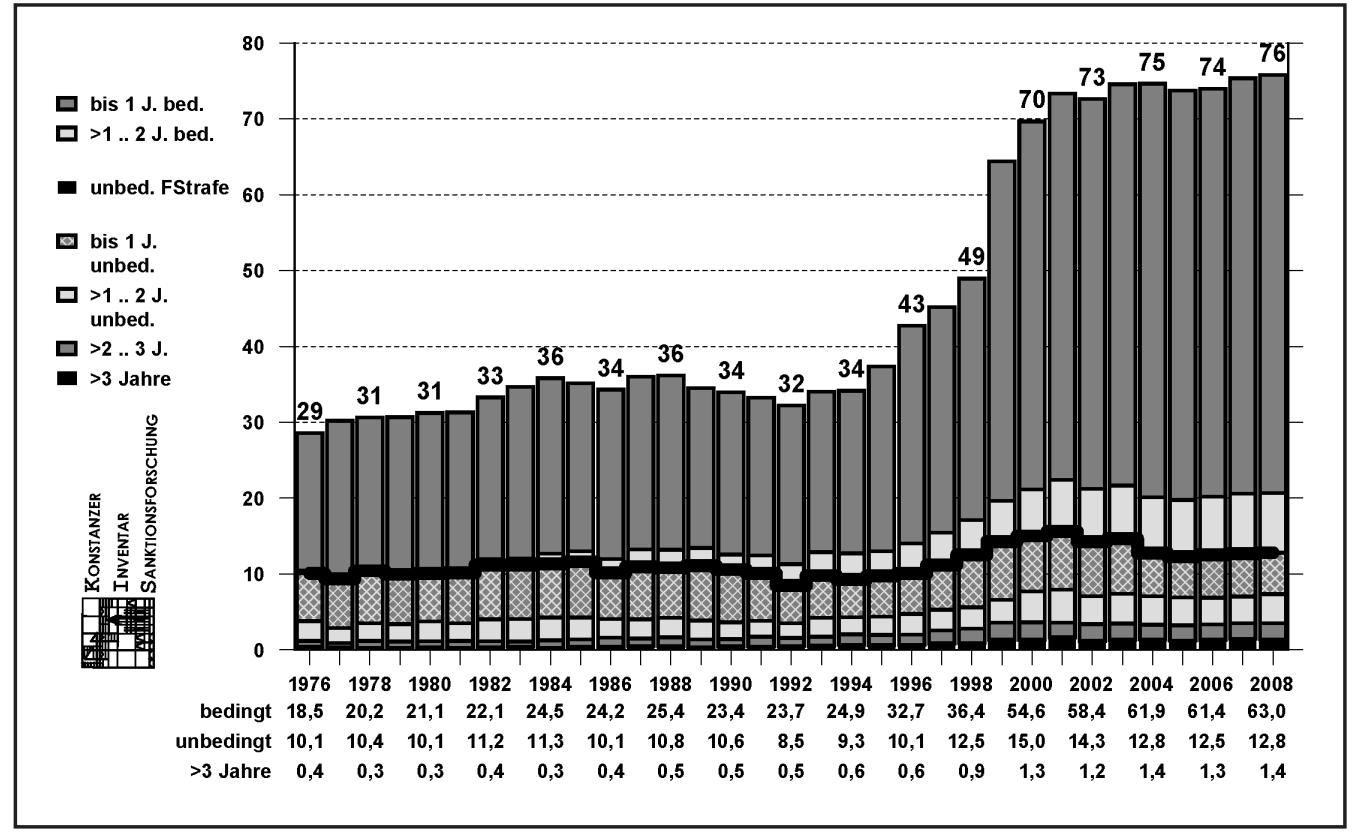

Datenquellen: Strafverfolgungsstatistik.

Im Unterschied hierzu weist die jugendstrafrechtliche Sanktionierungspraxis bei gefährlicher Körperverletzung ( $\$ 224$ StGB) - über den gesamten Zeitraum seit 1976 betrachtet - so gut wie keine Punitivitätstendenzen auf (vgl. Schaubild 11):

- Der Anteil der zu Jugendarrest oder Jugendstrafe Verurteilten ist - nach Rückgängen bis 1995 und Anstiegen bis 1998 - rückläufig und liegt deutlich unter dem Niveau der 1970er Jahre.

- Dieser Rückgang beruht fast ausschließlich auf der Sanktionierungspraxis bei Jugendarrest. Der Anteil der zu Jugendstrafe Verurteilten ist aber ebenfalls nicht gestiegen, sondern sogar leicht zurückgegangen.

- Der Anteil der zu unbedingter Jugendstrafe Verurteilten ist zwischen 1976 und 2008 im Wesentlichen unverändert geblieben. Wird freilich nur der Zeitraum 1990 bis 2008 betrachtet, dann sind wegen des Tiefstandes 1990 Zunahmen zu beobachten. Unter den unbedingten Jugendstrafen stieg der Anteil der längerfristigen Jugendstrafen von mehr als 2 Jahren geringfügig an.

- Bei den bedingten Jugendstrafen ging der Anteil der Jugendstrafen bis 1 Jahr ebenfalls zurück, Jugendstrafen zwischen 1 und 2 Jahren nahmen dagegen leicht zu.

- Während Jugendstrafen bis 1 Jahr seltener verhängt werden, werden Jugendstrafen zwischen 1 und 2 Jahren häufiger verhängt, und zwar als bedingte Jugendstrafen. Der Anteil der unbedingten Jugendstrafen dieser Kategorie ist indes nicht gestiegen. 
Schaubild 11: Wegen gefährlicher Körperverletzung ( $\$ 224$ StGB) zu Jugendarrest oder Jugendstrafe (insgesamt) Verurteilte nach der Strafdauer sowie Strafaussetzung zur Bewährung. Anteile bezogen auf Verurteilte. Früheres Bundesgebiet mit Westberlin, seit 2005 mit Gesamtberlin, seit 2007 Deutschland

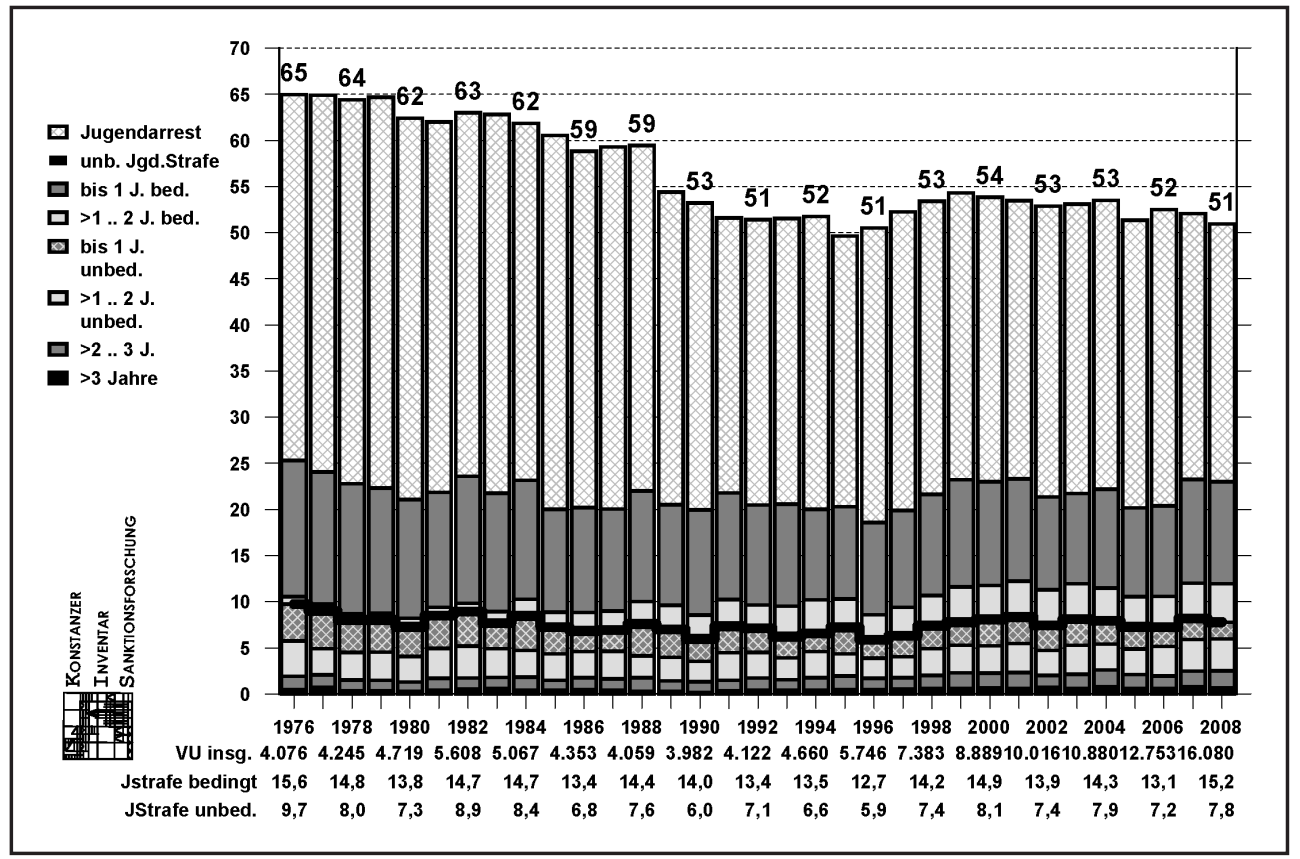

Datenquellen: Strafverfolgungsstatistik.

Bei Einbruchdiebstahl (\$S 243 I Nr. 2 StGB, seit 1998 auch $\$ 244$ I Nr. 3 StGB) zeigt sich (vgl. Schaubild 12):

- Der Anteil der zu Freiheits- oder Jugendstrafe Verurteilten ist leicht rückläufig, und zwar im Jugendstrafrecht stärker als im allgemeinen Strafrecht.

- Der Anteil der zu unbedingter Freiheits- oder Jugendstrafe Verurteilten ist zurückgegangen, der Anteil der zur Bewährung ausgesetzten Strafen ist dagegen gestiegen.

- Innerhalb der unbedingten Strafen hat sich das Strafenniveau nicht erhöht; der Anteil der ausgesetzten Strafen von mehr als 1 Jahr ist dagegen zugunsten der kürzeren Strafen etwas gestiegen.

Schaubild 12: Wegen Einbruchsdiebstahls ( $\$ \mathbb{S} 243$ I Nr. 2 StGB, seit 1998 auch $\$ 244$ I Nr. 3 StGB) zu Freiheits- oder Jugendstrafe Verurteilte nach der Strafdauer sowie Strafaussetzung zur Bewährung. Anteile bezogen auf Verurteilte. Früheres Bundesgebiet mit Westberlin, seit 2005 mit Gesamtberlin, seit 2007 Deutschland

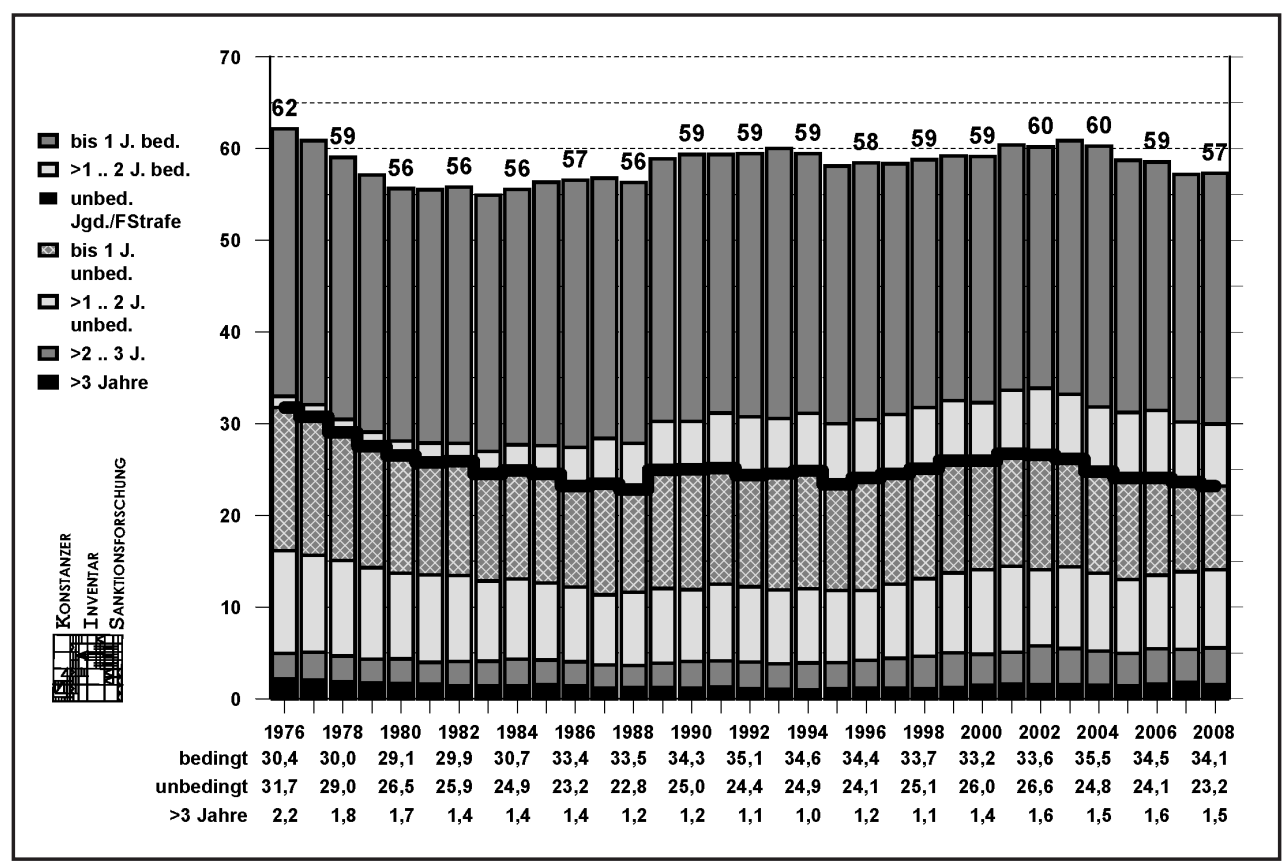

Datenquellen: Strafverfolgungsstatistik. 
Bei Raub (\$S 249, 250 StGB) findet sich ebenfalls keine empirische Stütze für die Punitivitätsthese (vgl. Schaubild 13):

- Der Anteil der zu Freiheits- oder Jugendstrafe Verurteilten ist deutlich rückläufig.

- Ebenso rückläufig entwickelt hat sich der Anteil der unbedingten Strafen.

- Innerhalb der unbedingten Strafen ging das Strafenniveau zurück.

- Lediglich innerhalb der bedingten Strafen kam es zu einer Verschiebung zugunsten der Strafen zwischen 1 und 2 Jahren.

Schaubild 13: Wegen Raubes $(\mathbb{S}$ 249, 250 StGB) zu Freiheits- oder Jugendstrafe Verurteilte nach der Strafdauer sowie Strafaussetzung zur Bewährung. Anteile bezogen auf Verurteilte. Früheres Bundesgebiet mit Westberlin, seit 2005 mit Gesamtberlin, seit 2007 Deutschland

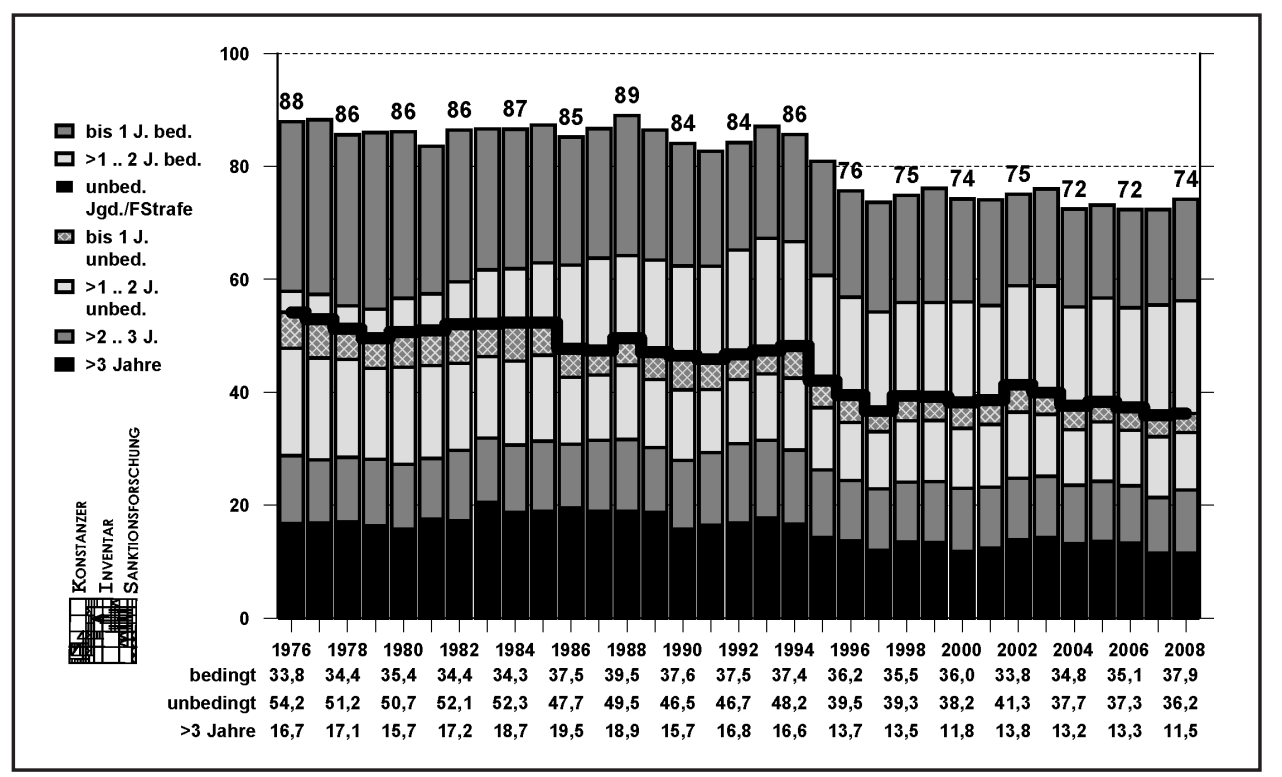

Datenquellen: Strafverfolgungsstatistik.

Die Aggregatdatenanalyse der schweren Kriminalitätsformen zeigt ein Bild, das vielfach als Doppelstrategie („bifurcation“) bezeichnet wird, als Verschärfung bei Gewalt- und Sexualdelikten (wobei auch dies in Deutschland nur eingeschränkt gilt), als Deinstitutionalisierung bei Eigentums- und Vermögenskriminalität. In der deutschen Sanktionierungspraxis ist diese „,bifurcation“ freilich nur schwach ausgeprägt. Insgesamt beschränken sich die Indikatoren für zunehmende Punitivität - jedenfalls bei einer Aggregatdatenanalyse - auf einen kleinen Bereich von weniger als $2 \%$ der Verurteilten.

Wenn dem so ist, dann bleibt freilich die Frage, weshalb die Zahl der Gefangenen - absolut wie bezogen auf 100.000 der strafmündigen Wohnbevölkerung - zugenommen hat (vgl. Schaubild 14).

Schaubild 14: Gefangene, Verwahrte, Untergebrachte (Stichtagszählung, jeweils 31.3.). Häufigkeitszahlen pro 100.000 der strafmündigen Wohnbevölkerung. Früheres Bundesgebiet mit Westberlin, seit 1992 (Gefangene, Sicherungsverwahrte) bzw. seit 1996 (Maßregelvollzug) mit Gesamtberlin

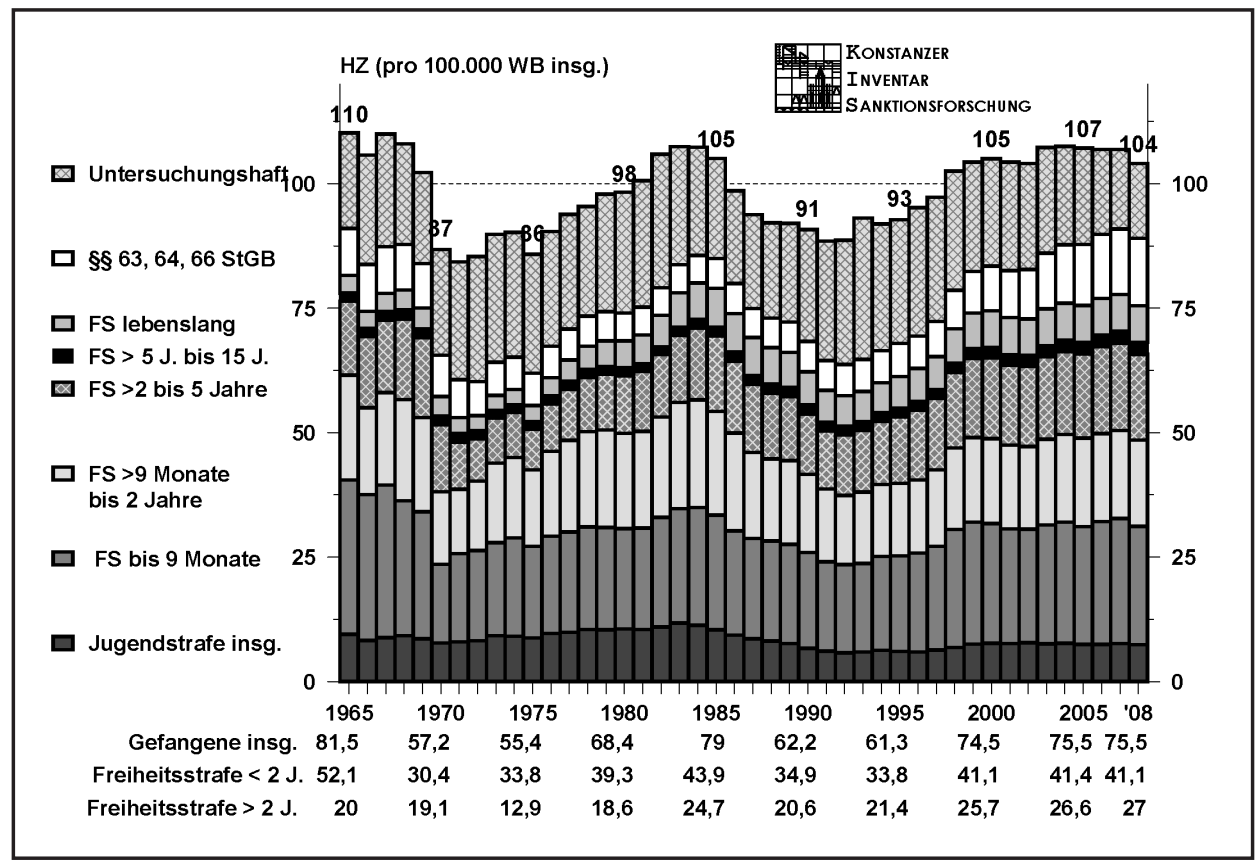

Datenquelle: Strafvollzugsstatistik: Maßregelvollzugsstatistik 
Zur Zahl der Gefangenen liegen in Deutschland nur Stichtagszahlen vor, die, wie eingangs erwähnt, eine Funktion der Zahl der Zugänge und der Verweildauer in den Anstalten sind. Der Einfluss der Sanktionierungspraxis auf die Gefangenenzahlen lässt sich nur zum Teil quantitativ bestimmen. Genaue Zahlen über die Zugänge, die auf gescheiterten Vollstreckungsverfahren (Ersatzfreiheitsstrafe) oder auf Widerrufen von Strafaussetzungen beruhen, fehlen. Die durchschnittliche Länge der zu unbedingter Freiheitsstrafe Verurteilten ist ebenso wenig bekannt wie die tatsächliche Vollstreckungsdauer (Strafrestaussetzung, Begnadigung, Abschiebung usw.). Möglich ist lediglich, den Einfluss zu bestimmen, den Veränderungen der Zuweisungsraten (unbedingte Freiheits- und Jugendstrafe) einerseits, Veränderungen der absoluten Zahlen der Verurteilten andererseits haben. Da zwischen 1990 und 2005 die Gefangenenraten angestiegen sind, sollen als Ausgangsgröße die Werte des Jahres 1990 zugrunde gelegt werden. Eine einfache Berechnung zeigt, dass der auf unbedingt verhängten Freiheits- und Jugendstrafen beruhende Zugang - insgesamt gesehen - nur zu 15\% auf einer Verschärfung der Sanktionierungspraxis, zu 85\% dagegen auf einem Anstieg der Verurteiltenzahlen beruht (vgl. Schaubild 15). Die deliktspezifische Analyse zeigt erneut die Doppelstrategie.

Schaubild 15: Zunahme unbedingt verhängter Jugend- und Freiheitsstrafen 1990. 2008 aufgrund von gestiegenen Verurteiltenzahlen oder einer Veränderung des Strafenniveaus. 1976 Früheres Bundesgebiet mit Westberlin, 2008 Deutschland

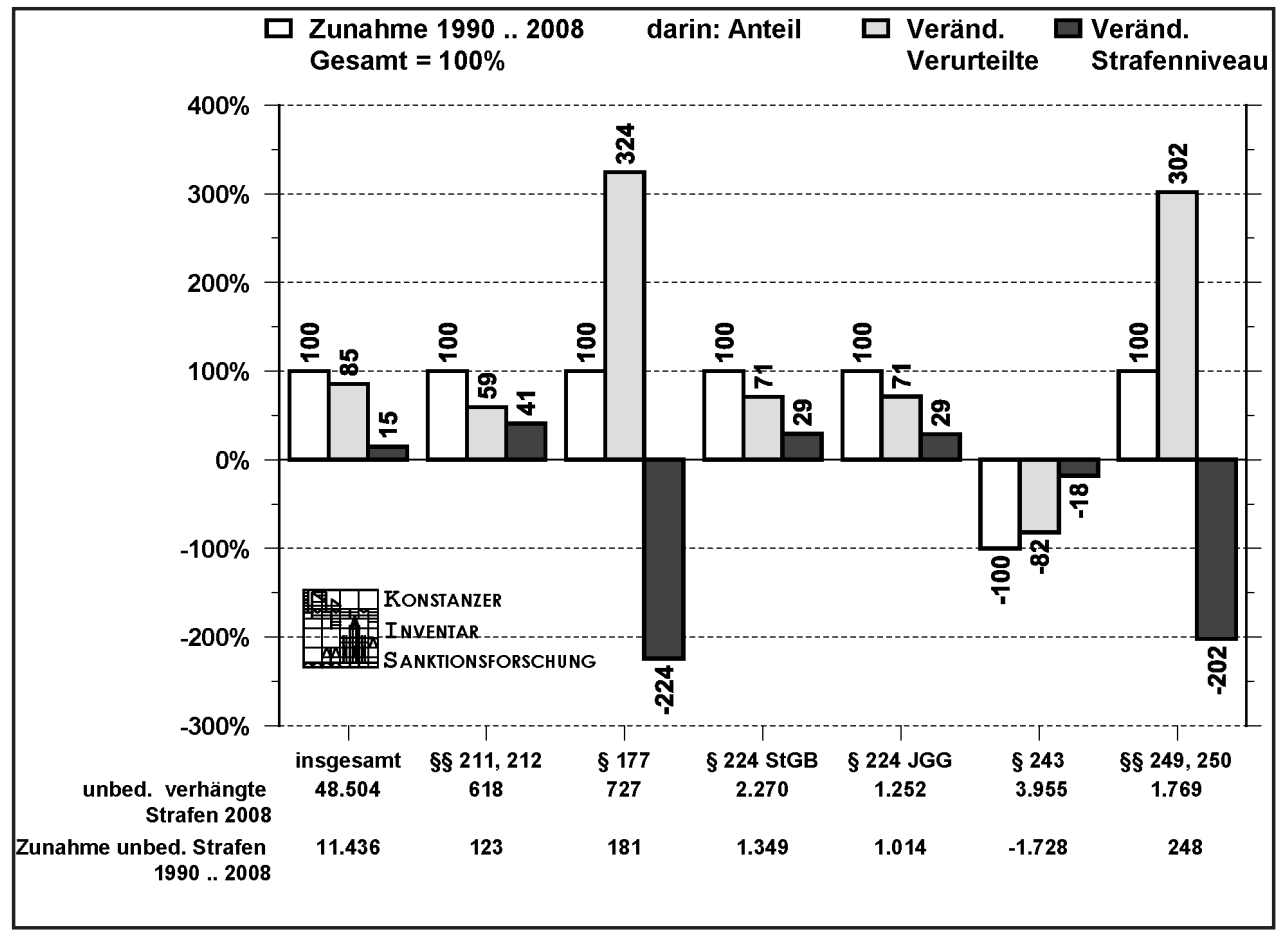

Datenquellen: Strafverfolgungsstatistik.

Erläuterung und Lesehilfe zum Schaubild am Beispiel Verbrechen und Vergehen insg.: 1990 erfolgten 692.363 Verurteilungen, davon 37.068 zu einer unbedingten Freiheits- oder Jugendstrafe (=5,4\%). 2008 wurden 874.691 Verurteilte registriert, davon waren 48.504 stationäre Sanktionen (= 5,5\%), es wurden also 11.436 stationäre Sanktionen mehr verhängt als noch 1990. Bei unveränderter Strafzumessung wären 2008 - wie 1990 - 5,4\% zu einer unbedingten Freiheitsstrafe verurteilt worden, also 46.830 statt der tatsächlichen 48.504 , d.h. dieses Plus (+1.674) beruht auf relativ häufiger erfolgter Verurteilung zu einer unbedingten Freiheits- oder Jugendstrafe. Daraus ergibt sich: Die Zahl der zu einer unbedingten Strafe Verurteilten ist zwar um 11.436 gestiegen, aber nur 14,6\% (1.674 in \% des Gesamtzuwachses von 11.436) beruhen auf verschärfter Strafzumessung, der Rest $(85,4 \%)$ ist schlicht Folge einer Zunahme der Verurteiltenzahlen

\subsection{Zunehmende Punitivität durch Verschärfungen bei der Strafvollstreckung?}

Punitivität wird immer wieder vermutet bei der Strafvollzugs- und Strafrestaussetzungspraxis. Punitivität als Ausdruck eines Niedergangs des Rehabilitierungsideals und gesteigerten Sicherheitsdenkens könnte zu einem Rückgang der im offenen Vollzug untergebrachten Gefangenen geführt haben.

Offener Vollzug ist empirisch die Ausnahme, nicht die Regel. Sowohl bei Erwachsenen, bei Heranwachsenden als auch bei Jugendlichen sind die Raten der im offenen Vollzug befindlichen Strafgefangenen in den letzten Jahren zurückgegangen. Freilich ist die Interpretation schwierig, ob es sich hierbei um eine punitive, dem Sicherheitsdenken geschuldete Tendenz handelt oder ob die Klientel schwieriger geworden ist. Denn die Strafvollzugspopulation hat sich nicht nur quantitativ, sondern vor allem qualitativ verändert, namentlich der Anteil der wegen Drogenoder Körperverletzung inhaftierten Gefangenen ist deutlich gestiegen. ${ }^{22}$

Zur Strafrestaussetzung, dem aussagekräftigsten Indikator, enthält die deutsche Strafvollzugsstatistik nur unzulängliche Daten. Seit 2003 wird die Zahl der „Abgänge“ aus dem Strafvollzug wegen Aussetzung des Strafrestes nur noch für die drei Stichtagsmonate (März, August, November) nachgewiesen. Diese Zahlen sind überdies, da auch Verlegungen in andere Anstalten als Abgang zählen, nicht personenbezogen. Schließlich ist die Bezugnahme auf die Zahl der Entlassungen (wegen Strafende oder Strafrestaussetzung) nicht möglich wegen der Vielzahl 
von Entlassungen aus Ersatzfreiheitsstrafe, bei denen eine Strafrestaussetzung rechtlich nicht zulässig ist. Die Strafrestaussetzungsrate lässt sich deshalb nicht valide bestimmen.

\subsection{Zunehmende Punitivität durch ein „Mehr“ an freiheitsentziehenden Maßregeln der Besserung und Sicherung?}

Als weiteres Indiz für zunehmende Punitivität kann schließlich die deutliche Zunahme der Unterbringungsanordnungen gemäß \$\$ 63, 64, 66 StGB gedeutet werden (vgl. Schaubild 16). Noch weitaus stärker als die im Mittelpunkt der Diskussion stehende Sicherungsverwahrung sind die Anordnungen gemäß $\$ \mathbb{S} 63,64$ StGB gestiegen. Freilich - es handelt sich in quantitativer Betrachtung um eine kleine Gruppe von 0,3\% (2008) der Abgeurteilten. Während die Unterbringung in einer Entziehungsanstalt nicht längerfristig ist ( $\$ 67 \mathrm{~d}$ I S. 1 StGB), können vor allem Sicherungsverwahrung sowie die Unterbringung in einem psychiatrischen Krankenhaus zu mehrjähriger Freiheitsentziehung führen. Fasst man deshalb alle Formen längerfristiger Freiheitsentziehung zusammen, also Freiheitsstrafe von mehr als 2 Jahren, Unterbringungen gemäß \$S 63, 66 StGB, dann sind hiervon 1,6\% der im Jahr 2008 Verurteilten betroffen.

Schaubild 16: Abgeurteilte mit Anordnung einer Unterbringung im psychiatrischen Krankenhaus, in einer Entziehungsanstalt oder in Sicherungsverwahrung. Anteile bezogen auf 1.000 Abgeurteilte. Früheres Bundesgebiet mit Westberlin, seit 1995 mit Gesamtberlin

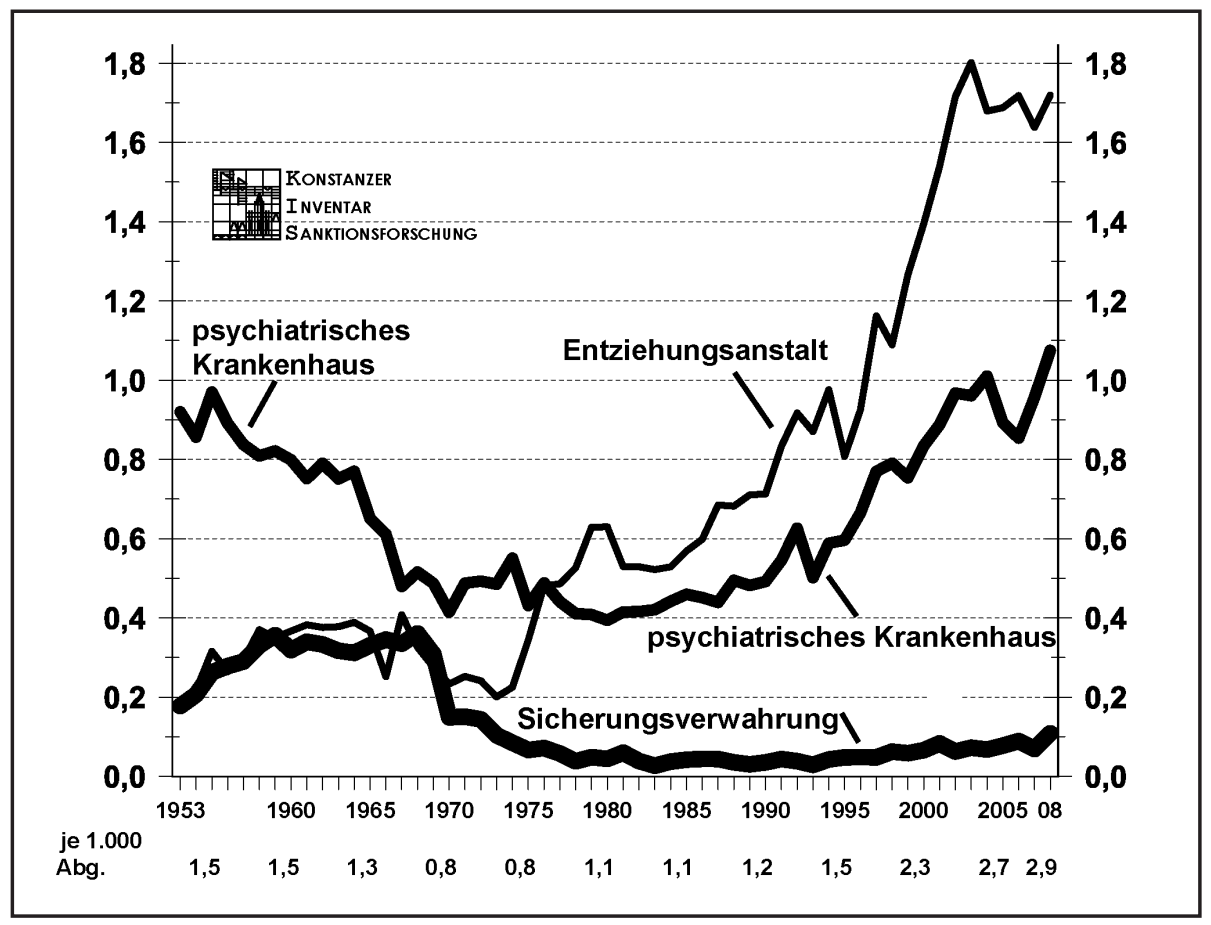

Datenquellen: Strafverfolgungsstatistik.

\section{Zusammenfassung}

Die empirische Prüfung, ob die deutsche Sanktionierungspraxis punitiver geworden ist, also mehr, härtere oder längere Strafen verhängt werden, ist aufgrund der Daten der Strafrechtspflegestatistiken methodisch nur unter einer Reihe von (unsicheren) Annahmen möglich.

Wird die gesamte Sanktionierungspraxis betrachtet, dann zeigt sich eine erstaunliche Konstanz. Weder im Bereich der staatsanwaltschaftlichen Erledigung von Ermittlungsverfahren noch im Bereich der Verurteilungen gibt es einen eindeutigen empirischen Beleg für ein „Mehr“ an Sanktionen.

Entgegen Annahmen der Punitivitätsthese sind die U-Haftraten nicht gestiegen und werden Heranwachsende nicht weniger häufiger in das JGG einbezogen.

Es gibt insgesamt auch keinen Beleg dafür, dass die formellen Sanktionen wesentlich härter geworden wären. Trotz Ausweitung der Diversionsentscheidungen ist der Anteil der stationären Sanktionen insgesamt nur leicht gestiegen. Der Anstieg des Anteils stationärer Sanktionen an den Verurteilten im Jahrzehnt zwischen 1992 und 2002 hat sich nicht fortgesetzt, sondern abgeschwächt. Überschätzt wird dieser Anstieg dadurch, dass als Folge zunehmender Diversion immer mehr leichte und mittelschwere Kriminalität gar nicht mehr zur Anklage kommt. Bezogen auf die Gesamtheit der Sanktionierten war jedenfalls der Anteil der stationären Sanktionen in den letzten Jahren weitgehend konstant; derzeit befindet er sich auf dem niedrigsten, jemals gemessenen Wert.

Der Anteil der zur Bewährung ausgesetzten Strafen ist deutlich gestiegen und blieb in den letzten Jahren im Wesentlichen unverändert.

Allerdings gibt es Erhöhungen des Strafenniveaus sowohl bei der Geldstrafe als auch im Bereich der unbedingt verhängten Freiheits- und Jugendstrafen. Bei der Geldstrafe dürfte es sich um eine Folge der zunehmenden Ausfilterung leichter und mittelschwerer Formen der Kriminalität durch Diversion handeln. Bei den stationären Sanktionen ist die Rate der zu Freiheitsentzug zwischen 2 Jahren bis einschließlich 5 Jahren Verurteilten gestiegen; bei Bezugnahme auf die (informell oder formell) Sanktionierten ist indes auch hier so gut wie keine Verschärfung feststellbar. Hiervon betroffen ist indes nur eine sehr kleine Gruppen der Verurteilten, denn nur bei 1,5\% der Verurteilten $(0,6 \%$ der Sanktionierten) wird ein derartiges Strafmaß ausgesprochen. 
Die Einzeldeliktanalyse zeigt gegenläufige Tendenzen - zunehmende Punitivität bei vorsätzlichen Tötungsdelikten und gefährlicher Körperverletzung (jedenfalls im allgemeinen Strafrecht), abnehmende Punitivität bei Eigentums- und Vermögensdelikten.

Deutliche Spuren haben hingegen die gesetzlichen Änderungen im Bereich der freiheitsentziehenden Maßregeln hinterlassen. Die Zahl der Unterbringungsanordnungen gem. $\$ \int 53,64,66 \mathrm{StGB}$ ist deutlich gestiegen. In rein quantitativer Betrachtung handelt es sich aber auch hier um eine relativ kleine Gruppe von 0,3\% der Abgeurteilten. Sicherung vor gefährlichen Tätern findet weitaus am Häufigsten durch Unterbringung im psychiatrischen Krankenhaus statt. 2008 waren 15mal mehr Personen gem. $\$ 63$ StGB untergebracht als gem. $\$ 66$ StGB.

Die Gefangenenraten sind zwischen 1990 und 2005 gestiegen. Bekanntlich sind diese Raten eine Funktion der Zahl der Zugänge und der Verweildauer. Mangels Daten zur durchschnittlichen Dauer der tatsächlich vollstreckten Freiheits- und Jugendstrafe lässt sich der Einfluss einer etwaigen Veränderung der Verweildauer nicht bestimmen. Dagegen lässt sich hinsichtlich der Zugangszahlen wegen unbedingt verhängter Freiheits- oder Jugendstrafen berechnen, welchen Einfluss einerseits eine Veränderung der Sanktionierungspraxis, andererseits eine Veränderung der Verurteiltenzahlen hat. Die Gegenüberstellung der Daten der Jahre 1990 und 2008 zeigt, dass - insgesamt gesehen - nur 15\% des Zuwachses an stationären Sanktionen auf einer veränderten Sanktionierungspraxis beruhten, $85 \%$ beruhen dagegen auf einer Zunahme der Verurteiltenzahlen.

Insgesamt heißt dies, dass die These von der „neuen Lust am Strafen“ für die deutsche Sanktionierungspraxis aufgrund der Aggregatdaten der Strafrechtspflegestatistiken empirisch nicht bestätigt werden kann. ${ }^{23}$ Nach den vorliegenden Daten handelt es sich bei der These von zunehmender Punitivität der deutschen Sanktionierungspraxis um einen zwar dem Zeitgeist entsprechenden, empirisch aber nicht hinreichend belegten Mythos. Richtig ist, dass es eine auf bestimmte, in quantitativer Hinsicht insgesamt sehr kleine Straftäter- und Deliktsgruppe beschränkte Tendenz zu mehr Punitivität gibt, die freilich teilweise sogar wieder rückläufig zu sein scheint. Es handelt sich einerseits um die Gruppe der als besonders „gefährlich“ eingestuften Täter sowie um Täter der Gewaltkriminalität. Für die weit überwiegende Mehrheit der informell oder formell Sanktionierten lassen sich jedoch keine wesentlichen Änderungen feststellen.

Dass die deutsche Sanktionierungspraxis dem punitiven Trend weitestgehend nicht erlegen ist, kann nicht nur mit dem traditionellen Konservatismus der Justiz erklärt werden. Die Wurzeln liegen eher im Rechtssystem selbst. Wo Richter und Staatsanwälte gewählt werden, wo der Einfluss der Laienrichter groß ist, ist der Einfluss der öffentlichen Meinung größer als in einem System mit professionellen, verbeamteten, unabhängigen Entscheidungsträgern und einem Laienrichtersystem, das die Entscheidungen über Schuld und Strafe nicht allein trifft und bei Kapitaldelikten in der stimmenzahlmäßigen Minderheit ist.

Prof. em. Dr. iur. Wolfgang Heinz, Lehrstubl für Kriminologie und Strafrecht, Universität Konstanz · Fachbereich Rechtswissenschaft, Rechts-, Wirtschafts-und Verwaltungswissenschaftliche Sektion, Universitätsstraße 10 · Fach D 119, D 78457 Konstanz, Telefon: (0)7531/882958/-2674·Telefax: (0)7531/88-4540,eMail:wolfgang.heinz@uni-konstanz.de, Web: http://www.jura.uni-konstanz.de/heinzl

\section{Fußnoten:}

1 Datenquelle: http://bjs.ojp.usdoj.gov/content/glance/corr2.cfm

$2 \mathrm{Zu}$ diesen und weiteren Verschärfungen vgl. vor allem Garland, David: Die Kultur der Kontrolle. Verbrechensbekämpfung und soziale Ordnung in der Gegenwart. Frankfurt/New York 2008, S. 261.

3 Vgl. Hofer, Hanns von: Anmerkungen zum Forschungsstand bzgl. der Erklärung von Gefangenenraten, in: Dünkel et al. (Hrsg.): Kriminalität, Kriminalpolitik, strafrechtliche Sanktionspraxis und Gefangenenraten im europäischen Vergleich, Mönchengladbach 2010, S. 25 ff.; Dünkel et al.: Gefangenenraten und Kriminalpolitik in Europa, in: Dünkel et al. aaO., S. 1030 ff.

4 Vgl. Schaubild 14.

5 Vorwort in Lautmann, R.; Klimke, D.; Sack, F. (Hrsg.): Punitivität. Kriminologisches Journal, 8. Beiheft, 2004, S. 4. Vgl. ferner zahlreiche Beiträge von Fritz Sack, zuletzt: Symbolische Kriminalpolitik und wachsende Punitivität, in: Dollinger, Bernd; Schmidt-Semisch, Henning (Hrsg.): Handbuch Jugendkriminalität. Kriminologie und Sozialpädagogik im Dialog, Wiesbaden 2010, S. 63 ff.

6 Im Parteimanifest 1997 von Labour hieß es „We will be tough on crime and tough on the causes of crime“(http://www.bbc.co.uk/election97/background/parties/manlab/labman6.html).

7 In der Koalitionsvereinbarung der rot-grünen Koalition von 1998 wurde die Labour-Formulierung als „Leitlinie“ für die „Innere Sicherheit“ übernommen: „entschlossen gegen Kriminalität und entschlossen gegen ihre Ursachen“ (http://www.spd.de/de/pdf/Koalitionsvertrag1998.pdf, IX).

8 „Was die Behandlung von Sexualstraftätern betrifft, komme ich mehr und mehr zu der Auffassung, dass erwachsene Männer, die sich an kleinen Mädchen vergehen, nicht therapierbar sind. Deswegen kann es nur eine Lösung geben: wegschließen - und zwar für immer“ (Alt-Bundeskanzler G. Schröder, Interview, „Bild am Sonntag“ vom 8.7.2001).

9 Lautmann, R.; Klimke, D.: Punitivität als Schlüsselbegriff für eine Kritische Kriminologie, in: Lautmann et al. (Anm. 5), S. 9.

10 Zum Jugendstrafrecht vgl. Heinz, Wolfgang: Aktuelle Entwicklungen in der Sanktionierungspraxis der Jugendkriminalrechtspflege, in: DVJJ (Hrsg.): Achtung (für) Jugend! Praxis und Perspektiven der Jugendkriminalrechtspflege, Tagungsdokumentation des 28. DJGT (im Druck); zum allgemeinen Strafrecht vgl. Heinz, Wolfgang: Neue Lust am Strafen. Gibt es eine Trendwende auch in der deutschen Sanktionierungspraxis?, in: Festschrift für Udo Ebert (im Druck).

11 Heinz, Wolfgang: Das strafrechtliche Sanktionensystem und die Sanktionierungspraxis in Deutschland 1882-2008. http://www.uni-konstanz.de/rtf/kis/Sanktionierungspraxis-in-Deutschland-Stand-2008.pdf, Schaubild 56.

$12 \mathrm{Zu}$ weiteren methodischen Problemen bei internationalen Vergleichen vgl. Dünkel et al. (Anm. 3), S. 1032 ff.; Dünkel/Morgenstern: Einführung, in: Dünkel et al. (Hrsg.) (Anm. 3), S. 6 f.

13 In der Bevölkerungsstatistik fehlen vor allem Touristen, Durchreisende, Berufspendler, sowie die zwar meldepflichtigen, aber nicht gemeldeten Personen, zum Beispiel illegal sich aufhaltenden Ausländer.

14 Vgl. Heinz, Wolfgang: Der schöne Schein des Strafrechts, Schaubild $2<$ http://www.uni-konstanz.de/FuF/Jura/heinz/Heinz_Schoener_Schein_StrafR.pdf>.

15 Vgl. Heinz (Anm. 14), Schaubild 4.

16 Untersuchungshaft wird in den Strafrechtspflegestatistiken lückenhaft und unvollständig nachgewiesen (vgl. Bundesministerium des Innern; Bundesministerium der Justiz (Hrsg.): Zweiter Periodischer Sicherheitsbericht, Berlin 2006 (http://www.bka.de/lageberichte/ps/psb2_langfassung.pdf), S. 550). Am vollständigsten sind die Daten der StVerfStat über die Zahl der Verurteilten mit vorangegangener Anordnung von U-Haft.

17 Vgl. Heinz (Anm. 11), Schaubild 47.

18 Unbedingte Freiheits- oder Jugendstrafe, unbedingter Strafarrest, Jugendarrest.

19 Als Indikator relevant ist nur die Anzahl der verhängten Tagessätze, die nach allgemeinen Strafzumessungsgrundsätzen bestimmt wird. Die Höhe der Tagessätze soll sich dagegen nach den wirtschaftlichen Verhältnissen des Verurteilten richten.

20 Gleichzeitig stiegen die Anteile der schuldunfähigen Abgeurteilten von 5 auf $12 \%$ bei Mord und von 10 auf $17 \%$ bei Totschlag, also nicht im selben Maße, um den Rückgang bei $\mathbb{} 21$ StGB auszugleichen. Für die Berechnung in Schaubild 9 ist dieser partielle Austausch zwischen $\mathbb{S} \$ 20,21$ StGB freilich unerheblich, weil nur auf die Verurteilten Bezug genommen wird, also ohne $\$ 20$ StGB.

21 Vgl. Dünkel/Morgenstern: Deutschland, in: Dünkel et al. (Hrsg.) (Anm. 3), S. 136, 216, mit einer weiter gefassten deliktischen Abgrenzung.

22 Vgl. Dünkel/Morgenstern (Anm. 21), S. 101.

23 Ebenso Dünkel/Morgenstern (Anm. 21); Dünkel et al. (Anm. 3), S. 1040. 